archives-ouvertes

\title{
Exegesis of Sect. III.B from "Fundamentals of the Mechanics of Continua" by E. Hellinger
}

\author{
Simon R. Eugster, Francesco Dell'Isola
}

\section{To cite this version:}

Simon R. Eugster, Francesco Dell'Isola. Exegesis of Sect. III.B from "Fundamentals of the Mechanics of Continua" by E. Hellinger. 2017. < hal-01568552>

\section{HAL Id: hal-01568552 \\ https://hal.archives-ouvertes.fr/hal-01568552}

Submitted on 25 Jul 2017

HAL is a multi-disciplinary open access archive for the deposit and dissemination of scientific research documents, whether they are published or not. The documents may come from teaching and research institutions in France or abroad, or from public or private research centers.
L'archive ouverte pluridisciplinaire HAL, est destinée au dépôt et à la diffusion de documents scientifiques de niveau recherche, publiés ou non, émanant des établissements d'enseignement et de recherche français ou étrangers, des laboratoires publics ou privés. 


\title{
Exegesis of Sect. III.B from "Fundamentals of the Mechanics of Con- tinua"* by E. Hellinger
}

\author{
Simon R. Eugster ${ }^{1,2 * *}$ and Francesco dell'Isola ${ }^{3,2}$ \\ ${ }^{1}$ Institute for Nonlinear Mechanics, University of Stuttgart, Stuttgart, Germany \\ 2 International Research Center for the Mathematics and Mechanics of Complex Systems, MEMOCS, Università \\ dell'Aquila, L'Aquila, Italy \\ ${ }^{3}$ Dipartimento di Ingegneria Strutturale e Geotecnica, Università di Roma La Sapienza, Rome, Italy
}

Received XXXX, revised XXXX, accepted XXXX

Published online XXXX

Key words Ernst Hellinger, elasticity, thermodynamics, electrodynamics, theory of relativity

This is our third and last exegetic essay on the fundamental review article DIE ALLGEMEINEN ANSÄTZE DER MECHANIK DER KONTINUA in the Encyklopädie der mathematischen Wissenschaften mit Einschluss ihrer Anwendungen, Bd. IV-4, Hft. 5 (1913) by Ernst Hellinger which contains the translation and the commentary of the remaining text starting from p. 663. The six subsections, No. 9-15, deal with the applications of the previously developed conceptual tools to formulate: an effective theory of elasticity, the dynamics of ideal fluids, models for internal friction and elastic hysteresis, a theory of capillarity, optics, the fundamental equations of electrodynamics, an introduction of the thermodynamical foundations and the relationship between the theory of continua and the theory of relativity. Hellinger refers to relevant literature while consolidating in an effective way the contemporary knowledge in 1913. Considering notational differences as being irrelevant for the characterization of the presented scientific content, Hellinger's article shows that an effective compendium of a large part of the insights given in Truesdell and Toupin [106] and Truesdell and Noll [105] has already been available in 1913. We include in this paper an assessment of the different roles played by pioneers, who are innovating their scientific discipline, and by erudite scholars whose role consists in re-ordering existent knowledge and advertising to a wider audience the most important technical results already obtained in a given discipline.

Copyright line will be provided by the publisher

\section{Introduction}

As in the previous papers $[42,43]$ of this exegetic series, the presented English translation of the original text written in German by Hellinger is typed in italic and is indented on both sides. The comments and explanatory remarks are in standard text style. We tried to produce a word-by-word translation totally refraining from allowing us to include any comment or interpretation of the original text because of translation. When we were obliged to introduce a word which had no correspondence in the German text we included it in square brackets as follows: [xxxx].

We want to start this third part of our exegesis with some general considerations motivated both by what we have read in Hellinger's contribution and by its apparent, mediated or hidden impact on subsequent literature. First of all, we want to state explicitly that we adopted a literal style in the translation for a precise reason. Indeed, we share the spirit and the intention of Willem van Moerbeke which can be considered as one of the founders of the technique of scientific translation, cf. the following subsection. Second, we will try to describe, by means of both more ancient and more modern (relevant in the present context) examples, some phenomena in the transmission of scientific knowledge whose importance is increasing in our period of fast and copious publication activity. Furthermore, also some issues concerning the establishment of priority in scientific innovation, the fair evaluation of preceding literature as well as the role of language and notational changes in the passage from one tradition to the other will be addressed. These issues are not raised in order to prove that some scientific ideas or theories were wrongly attributed, since in the perspective of scientific progress such a fact is not important at all. Actually, we are interested in the much more relevant problem how physical theories were formulated for the first time. This problem is of vital importance for instance when younger generations must be directed towards new scientific theories. It seems evident that the heuristic potential of variational methods, as shown by the history of science, imposes us to accept the central and preeminent role of variational methods. Finally, we will try to fairly assess the contribution of

* DIE ALLGEMEINEN ANSÄTZE DER MECHANIK DER KONTINUA. Encyklopädie der mathematischen Wissenschaften mit Einschluss ihrer Anwendungen. Bd. IV-4, Hft. 5 (1913).

** Corresponding author E-mail: eugster@inm.uni-stuttgart.de, Phone: +4971168568152 
some scholars whose role consisted either in re-ordering existent knowledge or in advertising the scientific results obtained by others. These scholars also contributed to that choral endeavor of scientific progress. However, one must be aware of the fact that on one side inventive pioneers came up with novel ideas (in general few ideas and in collaboration with many colleagues, cf. Archimedes' preface of "On the Method" quoted in the following sections) while, on the other side, erudite scholars re-organized bodies of knowledge which are so huge that it is not realistic to believe that they were conceived by a single person.

\subsection{Van Moerbeke's standard in the translation of scientific works}

In the Vatican Library, one can find an octavo manuscript on parchment called the "Ottobonianus Latinus 1850". This manuscript was rediscovered in the library repository, and its importance was fully understood, by the philologist Valentin Rose in 1881, [92]. The reasons for which the manuscript had disappeared, and the period during which no scholar could consult it, are all unknown. The second part of the aforementioned manuscript contains, among others, the manuscripts of the following outstanding works by Archimedes: On Spiral Lines, On Equilibrium of Planes (including the commentary of Eutocius), On the Measurement of the Circle, On the Sphere and the Cylinder (including the commentary of Eutocius), On Conoids and Spheroids, and On Floating Bodies. These masterpieces became then an important part of western science and have greatly influenced the Galilean Renaissance of modern mathematics and mechanics (for a detailed discussion the reader is referred to Clagett [16-19,21]. In [20], Clagett gives the proof that the translation found in this manuscript is due to Willem van Moerbeke (Gulielmus de Moerbecum ${ }^{1},{ }^{*} 1215-35-{ }^{\dagger} 1286$ ). Clagett established that it is in fact exactly Moerbeke's own copy, written in his own hand, and that it was made in 1269 at the papal court in Viterbo. Moerbeke apparently could consult two of the best Byzantine Greek manuscripts of Archimedes, both of which have since disappeared. In Byzantium, works of Archimedes have been studied without interruption until the sack occurring during the Fourth Crusade. Moerbeke has been a prolific medieval translator of philosophical, medical, and scientific texts from Greek language into Latin, during the period of Latin rule (following the conquer of Byzantium by the Fourth Crusade) of the Byzantine Empire. His translations were greatly influential at that time, when only few Archimedean Latin translations were available, and are still respected by modern scholars. Accordingly, they contributed to the diffusion of the Archimedean ideas during the Renaissance of the 12 th century. Moerbeke's translations became standard classics already in the 14 th century. ${ }^{2}$

In the translation presented here, we have chosen to follow strictly the standard established by Moerbeke. We did not try to produce elegant English, and maybe we could not attain such a result anyway, but we have translated the German word and sentences moulding them on the original German. Being both English and German two Anglo-Saxon languages strongly influenced by Latin, we believe that this was the right choice. In the opinion of Clagett $[18,20]$ and Heiberg [64,65], Moerbeke did understand well enough the mathematics by Archimedes to be able to correct many corrupted formulas in the manuscript which he was translating. Actually they believe that Moerbeke failed to master only some of the most sophisticated mathematical passages which he was translating. However, this literal style allowed Heiberg to reconstruct the Archimedean original without problems. The vicissitudes of Archimedes' masterpieces should not surprise the reader. One should consider that the here translated article by Hellinger has almost fallen into oblivion in a period where storage of information is much more efficient.

\subsection{Pioneers vs. Colonist: their different role in production and transmission of knowledge}

The content of this section can be resumed by a slight modification of the following quotation by André Gide.

«Everything that needs to be said has already been said. But since no one was listening, everything must be said again.»

Since we believe in the "average" (in space and time) increase of human knowledge, we would replace "Everything" with "Nearly everything". On the other hand we must accept regretfully that regressions did occur often and therefore that they may occur again. Although many efforts have been done in the history of natural philosophy in this direction, up to now only a rather superficial understanding has been reached in the development of the theory of recurrence of social phenomena. A rather exhaustive account of the more ancient development of the treated ideas can be found in Trompf [101] and a second volume on the subject has been announced by the same author. The general perception of historical phenomenology as described by Trompf leads to the individuation of some positive cycles ("corso" in the nomenclature by Giambattista Vico) followed by a regressive cycle ("ricorso") and then eventually again by a "corso". Vico decisively denies the conception

\footnotetext{
1 He is known in the English speaking world as William of Moerbeke, a Dominican by vocation, Latin Archbishop of Corinth from 1277 until his death 1286 and a papal penitentiary and chaplain (1265-1278). His date of birth is not documented accurately.

2 Henricus Hervodius could easily assess their enduring value: they were, at the same time, literal (de verbo in verbo), faithful to the spirit of the Greek original text and without elegance. See http://www.worldlibrary.org/articles/translations_into_latin_(c._1050-c.1250)
} 
of a historical evolution which monotonously increases by developing towards the progress and advancement of human societies. It seems (see again Trompf $[101,102]$ ) that Vico's work "Principj di Una Scienza Nuova" (Principles of a New Science) is not the first influential work and theoretical effort in the study of the recursive structure of social phenomena. In this context, one definitely has to mention the still-now-available parts of the Histories by Polybius (see e.g. [79]). Instead of the terms "corso" and "ricorso", Polybius used the term "anacyclosis" ( $\alpha \nu \alpha x \dot{\nu} x \lambda \omega \sigma \iota \varsigma)$ meaning literally "back-cycle" or "repeated cycle". The Italian word "ricorso" is a wise linguistic mold of "anacyclosis". Paraphrasing Vico or Polybius in the context of history of science, we want to try to individuate a specific "anacyclosis".

The scientific pioneer, never alone but supported by a creative and advanced scientific milieu, formulates groundbreaking theories. Usually, these theories are complex and require some technical knowledge which is not widely diffused. These theories are more or less readily accepted by the immediate scientific neighborhood of the pioneer and often applied and developed further by its members. For several reasons a loss and or partial erasure of the original insights may occur during the transmission of these theories. The reasons can be, among others, economical and social crises and decay, the change in the language of transmission or the loss of social consideration of the importance of science. After a while, when the social conditions change, the ancient theory can eventually be slowly recovered. The recovery occurs via several efforts, including more or less independent reformulations partially based on corrupted informations about the original complete version of the theory but also based on novel and original understanding of the ancient ideas. Sometimes (like in the case of Tartaglia's ${ }^{3}$ translation of Archimedes works) the modifications to the old theory dramatically decrease the quality of the presentation, which still may remain basically correct, while in other cases (as we will see in the case of Galilean reformulation of the basic ideas of mechanics) these modifications start new flows of research and initiate new advancements of knowledge. This is the situation in which scientific knowledge seems to appear "out of the blue" and often the naive explanation is the presumed "geniality" of a single scientist who could alone formulate enormous bodies of knowledge. Actually, the "rediscoveries" are made possible by the erudition of "colonist" scientists, who, following the directions of the pioneers, arrive in the "Newfoundland" and start to systematically explore it, by carefully and effectively describing their results in the textbooks. They often pretend to be pioneers by hiding or underestimating the importance of the heritage they received from the pioneers. The reader is referred to Russo [93] for a more detailed discussion about the actual appearance of this specific "anacyclosis".

Here we want to describe a first example showing how original results dating up to Archimedes of Syracuse resurfaced slowly until very recent historical periods. The detailed reconstruction of the relevant series of events was very difficult and needed the joint efforts of many philologists. However, we will describe also a second example, where Hellinger's article, which we are translating here, played a relevant role. The details of the corresponding "anacyclosis" can be (and will be) much more easily reconstructed, as all sources are easily available. Archimedes of Syracuse has been beyond all doubt one of the greatest if not the greatest scientist of the Hellenistic period. The reader is referred to the many works reconstructing the tormented history of the "lost and found" of his works in their travel in space (Alexandria, Jerusalem, North Africa, Arabic Peninsula, Rome, Venice, Florence, Constantinople, Paris, USA), in time (since the 3rd century B. C. until nowadays) and in between different languages (Doric Greek, Arabic, Latin, Italian, French, German and English), cf. $[37,64,65,76,77]$. Available ancient sources agree to consider Archimedes the most complete scientist of his period and probably for this fame many of his works were hand-copied and then printed so that in the end they were transmitted to us. This is proven, for instance, by the following excerpt from Pappus being identified by Thomas as the last Hellenistic mathematician. On pp. 614-621 of [99], one can read the translation given by Ivor Thomas. ${ }^{4}$

«The science of mechanics, my dear Hermodorus, has many important uses in practical life, and is held by philosophers to be worthy of the highest esteem, and is zealously studied by mathematicians, because it takes almost first place in dealing with the nature of the material elements of the universe. For it deals generally with the stability and movement of bodies [about their centers of gravity], and their motions in space, inquiring not only into the causes of those that move in virtue of their nature, but forcibly transferring [others] from their own places in a motion contrary to their nature ; and it contrives to do this by using theorems appropriate to the subject matter. The mechanicians of Heron's school say that mechanics can be divided into a theoretical and a manual part ; the theoretical part is composed of geometry, arithmetic, astronomy and physics, the manual of work in metals, architecture, carpentering and painting and anything involving skill with the hands. The man who had been trained from his youth in the aforesaid sciences as well as practiced in the aforesaid arts ${ }^{5}$, and in addition has a versatile mind, would be, they say, the best architect and inventor of mechanical devices. But as it is impossible for the same person to familiarize himself with such mathematical studies and at the same time

\footnotetext{
3 Nicolò Fontana Tartaglia * 1499/1500 - ${ }^{\dagger} 1557$ was an Italian mathematician, engineer, surveyor and a bookkeeper from the then-Republic of Venice.

4 To underline some sentences in the upcoming quotations, we printed them in bold. Otherwise, we tried to keep the typography as original as possible.

5 We prefer to translate the Greek word technas with techniques.
} 
to learn the above-mentioned arts, they instruct a person wishing to undertake practical tasks in mechanics to use the resources given to him by actual experience in his special art. ${ }^{6}$

Of all the [mechanical] arts the most necessary for the purposes of practical life are : (1) that of the makers of mechanical powers, they themselves being called mechanicians by the ancients - for they lift great weights by mechanical means to a height contrary to nature, moving them by a lesser force ; (2) that of the makers of engines of war, they also being called mechanicians - for they hurl to a great distance weapons made of stone and iron and such-like objects, by means of the instruments, known as catapults, constructed by them ; (3) in addition, that of the men who are properly called makers of engines — for, by means of instruments for drawing water which they construct water is more easily raised from a great depth ; (4) the ancients also describe as mechanicians the wonder-workers, of whom some work by means of pneumatics, as Heron in his Pneumatica, some by using strings and ropes, thinking to imitate the movements of living things, as Heron in his Automata and Balancings, some by means of floating bodies, as Archimedes in his book On Floating Bodies, or by using water to tell the time, as Heron in his Hydria, which appears to have affinities with the science of sun-dials ; (5) they also describe as mechanicians the makers of spheres, who know how to make models of the heavens, using the uniform circular motion of water.

Archimedes of Syracuse is acknowledged by some to have understood the cause and reason of all these arts ; for he alone applied his versatile mind and inventive genius to all the purposes of ordinary life, as Geminus the mathematician says in his book On the Classification of Mathematics. Carpus of Antioch says somewhere that Archimedes of Syracuse wrote only one book on mechanics, that on the construction of spheres, not regarding any other matters of this sort as worth describing. Yet that remarkable man is universally honored and held in esteem, so that his praises are still loudly sung by all men, but he himself on purpose took care to write as briefly as seemed possible on the most advanced parts of geometry and subjects connected with arithmetic; and he obviously had so much affection for these sciences that he allowed nothing extraneous to mingle with them. Carpus himself and certain others also applied geometry to some arts, and with reason; for geometry is in no way injured, but is capable of giving content to many arts by being associated with them, and, so far from being injured, it is obviously, while itself advancing those arts, appropriately honored and adorned by them.»

Furthermore, Archimedes knew exactly his place in history of science, as it is clearly indicated by the following excerpt in which one can hear his true and strong voice being directed to us (who try to deserve to be called his successors). Every time when we read this text, we reinforce our belief that Archimedes (and all his Hellenistic colleagues) are modern scientists. Surely more modern than all his epigones in the Middle Ages and as modern as for instance Lagrange, Cauchy or Navier. The original Greek source, we refer to, together with its Latin translation can be found in [65] between p. 426 line 3 and p. 430 line 22. The following excerpt of the English translation of Archimedes" "The Method" is from Heath [62] pp. $12-14$

«Archimedes to Eratosthenes greeting.

I sent you on a former occasion some of the theorems discovered by me, merely writing out the enunciations and inviting you to discover the proofs, which at the moment I did not give. The enunciations of the theorems which I sent were as follows.

[...] The proofs then of these theorems I have written in this book and now send to you. Seeing moreover in you, as I say, an earnest student, a man of considerable eminence in philosophy, and an admirer [of mathematical inquiry], I thought fit to write out for you and explain in detail in the same book the peculiarity of a certain method, by which it will be possible for you to get a start to enable you to investigate some of the problems in mathematics by means of mechanics. This procedure is, I am persuaded, no less useful even for the proof of the theorems themselves; for certain things first became clear to me by a mechanical method, although they had to be demonstrated by geometry afterwards because their investigation by the said method did not furnish an actual demonstration. But it is of course easier, when we have previously acquired, by the method, some knowledge of the questions, to supply the proof than it is to find it without any previous knowledge. This is a reason why, in the case of the theorems the proof of which Eudoxus was the first to discover, namely that the cone is a third part of the cylinder, and the pyramid of the prism, having the same base and equal height, we should give no small share of the credit to Democritus who was the first to make the assertion with regard to the said figure though he did not prove it. I am myself in the position of having first made the discovery of the theorem now to be published [by the method indicated], and I deem it necessary to expound the method partly because I have already spoken of it and I do not want to be thought to have uttered vain words, but equally because I am persuaded that

\footnotetext{
6 This is already the beginning of the engineering specialization.
} 
it will be of no little service to mathematics; for I apprehend that some, either of my contemporaries or of my successors, will, by means of the method when once established, be able to discover other theorems in addition, which have not yet occurred to me.

First then I will set out the very first theorem which became known to me by means of mechanics, namely that:

Any segment of a section of a right-angled cone (i.e. a parabola) is four-thirds of the triangle which has the same base and equal height,

and after this I will give each of the other theorems investigated by the same method. Then, at the end of the book, I will give the geometrical [proofs of the propositions]...»

In the "Quadrature of a Parabola", Archimedes writes what is translated by Heiberg in [65] between page 262 line 2 and page 266 line 4 into Latin and by Thomas [99], pp. 229-233 into English:

«Archimedes to Dositheus greetings.

On hearing that Conon, who fulfilled in the highest degree the obligations of friendship, was dead, but that you were an acquaintance of Conon and also versed in geometry, while I grieved for the death of a friend and an excellent mathematician, I set myself the task of communicating to you, as I had determined to communicate to Conon, a certain geometrical theorem, which had not been investigated before, but has now been investigated by me, and which I first discovered by means of mechanics and later proved by means of geometry. Now some of those who in former times engaged in mathematics tried to find a rectilineal area equal to a given circle and to a given segment of a circle, and afterwards they tried to square the area bounded by the section of the whole cone and a straight line, assuming lemmas far from obvious, so that it was recognized by most people that the problem had not been solved. But I do not know that any of my predecessors has attempted to square the area bounded by a straight line and a section of a right-angled cone, the solution of which problem I have now discovered; for it is shown that any segment bounded by a straight line and a section of a right-angled cone is four-thirds of the triangle which has the same base and height equal to the segment, and for the proof this lemma is assumed : given [two] unequal areas, the excess by which the greater exceeds the less can, by being added to itself, be made to exceed any given finite area. Earlier geometers have also used this lemma : for, by using this same lemma, they proved that circles are to one another in the duplicate ratio of their diameters, and that spheres are to one another in the triplicate ratio of their diameters, and also that any pyramid is a third part of the prism having the same base as the pyramid and equal height ; and, further, by assuming a lemma similar to that aforesaid, they proved that any cone is a third part of the cylinder having the same base as the cone and equal height. In the event, each of the aforesaid theorems has been accepted, no less than those proved without this lemma ; and it will satisfy me if the theorems now published by me obtain the same degree of acceptance. I have therefore written out the proofs, and now send them, first as they were investigated by means of mechanics, and also as they may be proved by means of geometry. By way of preface are included the elements of conics which are needed in the demonstration. Farewell.»

Here some remarks are required. Firstly, the Archimedes' style of writing is as Tacitean as the style of Hellinger. Secondly, Archimedes is very careful in respecting the works of «those who in former times engaged in mathematics» and he checks carefully that «it was recognized by most people that the problem had not been solved». Moreover, he claims that: «But I do not know that any of my predecessors has attempted to». Respecting the contribution of predecessors is fundamental in science. Not only one is obliged to review that some results have not been obtained already, but also one has to check that the whole scientific ambient agrees with such a statement. Finally, one more technical observation is of interest. In the second part of this exegetic series, we have seen how Hellinger describes the asymptotic expansion procedures leading to higher gradient three-dimensional theories and to the theory of plates and beams. Hellinger is a too sophisticated mathematician to believe that these asymptotic expansions are a "true" mathematical proof of the convergence of one theory to another. As the article deals with "mechanics" he describes the heuristic procedure leading to "the conjecture" of a mathematical result. Exactly as done by Archimedes, modern mathematicians exploited these conjectures to state a theorem to be proven with a mathematical rigorous technique. One among the most popular techniques which are used in this context is the theory of Gamma-convergence, due to De Giorgi. The ancient "method" described by Archimedes is faithfully followed by his successors. One of the most popular translation into Latin of Archimedes' works is signed by Nicolò Tartaglia. The title of its Translation (1543) reads in Latin as follows:

Opera Archimedis Syracusani Philosophi et Mathematici ingeniosissimi per Nicolaum Tartaleam Brixianum (Mathematicarum scientiarum cultorem) multis erroribus emendata, expurgata, ac in luce posita, multisque necessariis additis, quae plurimis locis intellectu difficillima erant, commentariolis sane luculentis et eruditissimis 
aperta, explicata atque illustrata existunt. Appositisque manu propria figuris quae graeco exemplari deformatae ac depravatae erant, ad rectissimam Symetriam omnia instaurata, reducta et reformata elucent. ${ }^{7}$

First of all, the reader should remark that in the aforementioned text it is not clear if Tartaglia declares to have corrected only the corrupted Greek manuscript in his hands or if he declares actually to have improved the very oeuvres by Archimedes, improving the Archimedean presentation of the results.

In order to understand the true role of Tartaglia in the transmission of Archimedes' work, as we lack the required competences in Doric Greek and Latin, we needed to resort (again an effect of linguistic barriers) to English or German sources. The statements which will follow are proven in the works by Heiberg [64, 65] (whose Latin Prolegomena we could very slowly interpret in some parts: we believe that because of their importance they should be completely translated into English), Heath [62], Clagett [16-19,21] and Dijksterhuis [37]. The reader is referred to them for the scholarly and philologically correct description of the evidence leading to all conclusions which we resume here. Remark that the cited authors are regarded as the greatest experts about the works by Archimedes ever active.

The oldest printed form of the works of Archimedes, we are aware of, is given by a now very rare work by Luca Gaurico "On the quadrature of the circle" published in Venice in 1503, [37]. This copy contains the Latin translation of the "Measurement of the Circle and Quadrature of the Parabola" as taken from Codex $\mathfrak{B}^{8}$ before it was corrected by Andreas Conerus ${ }^{9}$, cf. Heiberg $[64,65]$. A literal copy of this edition, enlarged with the Latin text of "On the Equilibrium of Planes" and of the first book of "On Floating Bodies", appears inside the work published in 1543 by Nicolò Tartaglia, [37]. Here we cite verbatim Dijksterhuis [37], pp. 40-41:

«In his preface the editor boasts of the great difficulties which he had to overcome in the deciphering and the translation of ancient and all but illegible Greek manuscripts, and which he only managed to surmount through his "incredible desire" to accomplish the work. According to Heiberg, however, it is a barefaced lie that he used any Greek text at all; he merely copied the edition of Gaurico and a copy of Codex B, errors and all.»

Indeed, the part of Tartaglia's translation which is not a literal copy from Gaurico has been proven by Clagett to be "dependent" on Moerbeke's translation. Concerning the part of "On Floating bodies" we cite again verbatim Dijksterhuis [37], p. 425:

«[...], lacking in [Codex] A, Heiberg resorted primarily to the Latin version by Tartaglia (1543), which he thought was based on a lost Greek manuscript (see Heiberg 1880-81, II:359n]), but which Clagett [1978, 553-56 ${ }^{10}$ has since shown depended on Willem of Moerbeke's Latin translation (1269). After the appearance of his edition, Heiberg came upon the Moerbeke translation and established that, while it for the most part reflected the A tradition, in several of the mechanical writings it referred to a second manuscript. The Latin (designated B) was so literal in style that Heiberg could use it as further evidence of the text. Later, through an extraordinary find, Heiberg identified the Archimedean contents of a certain tenth-century palimpsest manuscript and inspected it at Constantinople in 1906. This text (designated C) provided a new line of the text; not only did it compensate for scribal deficiencies of A and offer practically the whole of [the work On Floating Bodies], but also it preserved most of the text of the Method, unique in its witness to Archimedes' heuristic techniques.»

However, again citing Dijksterhuis [37], p. 439:

«The two books of Floating Bodies — particularly the first on general principles of hydrostatics - were influential in the Renaissance through the Latin versions of Tartaglia and Commandino ${ }^{11}$, and spawned numerous adaptations and commentaries, such as those of Stevin, Guidobaldo, and Galileo. For texts and discussion, see Dijksterhuis [1955; 1970]; Drake and Drabkin [1969]; Clagett [1978]; Drake [1981]. ${ }^{12}$

7 This title could be translated as follows: «The Oeuvres by Archimedes of Syracuse, very ingenious philosopher and mathematician, corrected and purified by many mistakes and enlightened by Nicolò Tartaglia (scholar of mathematical sciences) from Brescia, which in many places were very difficult to understand, are presented [being] opened, explained and illustrated by many added, really wide and very erudite and necessary commentaries. These [oeuvres] with the apposition by his own hand of pictures which, in the Greek exemplar, were deformed and corrupted, [now] shine in the totality reduced, reshaped and reestablished to perfect symmetry.»

8 In the Greek speaking scientific world at least three different codices, i.e. a bound collection of works, containing Archimedes' works existed: Codex A, Codex $\mathfrak{B}$ and Codex C. Codex A was preserved due to Lorenzo de Medici, Codex $\mathfrak{B}$ is available mainly due to its translation into Latin by de Moerbeke and for what concerns the rocambolesque fate of Codex C, the reader should consult [77].

9 The work of this scholar is unknown to the great majority of Archimedean students. In [37] on pp. 38, it is stated that «Codex B itself was in the possession of the German priest Andreas Conerus $(\dagger 1527)$ in 1508 in Rome; he took great interest in Greek mathematics and made several corrections in the text. Who were the other owners up to the year 1740, when it got into the library of the Vatican, is to be found accurately enumerated in Heiberg (Opera III, lxiii)»

${ }^{10}$ Heiberg 1880-81 and Clagett 1978 correspond to the references $[18,63]$ in the paper at hand.

11 For biographical details about Federico Commandino cf. http://www-history.mcs.st-andrews.ac.uk/ history/Biographies/Commandino.html.

12 The cited references correspond to $[18,35,36,38,39]$ in the paper at hand. 
Therefore undoubtedly the "propaganda" of the Archimedean works due to Tartaglia has the merit to have made possible the renaissance of hydrostatics (even if the true translation of the original work is most likely due to Gaurico and De Moerbeke). Moreover, it has been questioned recently (also after the rediscovery of Archimedes' palimpsest, see Netz and Noel [77] the role of Archimedes' oeuvres in the modern (re-)discovery of

i) the theory of real numbers via sections of rational numbers (so called Dedekind's section),

ii) the theory of Riemann integrals and

iii) the principle of virtual work in mechanics (see also Vailati [112]).

In our opinion the modern versions of these theories and principles are simply a reformulation of what modern scientists have learnt (directly or indirectly) from Hellenistic mathematical texts and in particular from Archimedes' works. Of course we have no reason to attribute the original discovery of them to Archimedes. He simply was aware of them. He used the results of his predecessors.

The second example we want to evoke here more directly concerns the theory which Hellinger calls "Mechanics of Continua". Hellinger does not attribute to himself any of the results he his presenting, even if his use of the method of Lagrange multipliers is so innovative that (see our comments in the second paper of the series) Reissner tried to prove that Hellinger did not formulate what is now called the Hellinger-Reissner variational principle. In his article, Hellinger carefully recognizes the role of his predecessors, even if, in our opinion, he could not faithfully attribute to Piola many contributions, cf. [23]. Hellinger is, exactly as Piola, a follower of Lagrange. His "Mechanics of Continua" is all based on the principle of virtual work. In the present exegesis, all ancillary theories, deduced from the (meta)-theory (i.e. continuum mechanics) are elegantly framed in a unified way. This is exactly what has been done by Truesdell, Toupin and Noll $[105,106]$. Among them, unfortunately, only Toupin believed in the central role of the principle of virtual work [personal communication Montreal 2013]. Both Truesdell and Noll believed, instead, in the more involved axiomatization based on the postulation of balance laws. The need of developing a general conceptual frame, i.e. continuum mechanics, in which all particular theories find their place under further assumptions is understood by Hellinger, who conceived the article translated here to exhibit the economy of thought obtained by assuming such a point of view. It is not easy to establish if Hellinger was the first scientist developing such a vision: it is sure, however, that his works preceded the monumental textbooks of Truesdell and Toupin [106] as well as Truesdell and Noll [105]. Unfortunately, only in the first of them a very limited discussion about variational principles is included, simply for criticizing them rather bitterly and in which Hellinger is cited as an advocate of the postulation of continuum mechanics based on the principle of virtual work. However, we believe that Hellinger's presently translated work did suggest to Truesdell the unifying vision of a field theory incorporating all more particular ones. In Hellinger's article these are:

i) A theory of elasticity (No. 9), which is developed in the fully nonlinear case, taking into account the objectivity requirements of constitutive equations; also beams and plates are considered;

ii) The dynamics of ideal fluids (No. 10), which is presented based on the results of D'Alembert and Piola including the case of incompressible fluids;

iii) A theory of internal friction and elastic hysteresis (No. 11), which is presented by introducing the Rayleigh potential for internal friction; furthermore Boltzmann memory effects are considered together with the basic ideas of plasticity and its potential to the description of sandy materials;

iv) A theory of capillarity (No. 12), including a treatment of the Laplace concept of surface energy with the suggestion of some generalizations which are even nowadays not completely explored;

v) Optics (No. 13), showing its structure as a field theory, ready to establish its relationship with electrodynamics;

vi) Electrodynamics (No. 13), in which the nature of free ether is considered and the structure of the field theory leading to Maxwell's equations is described;

vii) Thermodynamics (No. 14), where one believes to read many parts of the models subsequently developed by Coleman and Noll;

viii) The theory of relativity (No. 15), in which a variational formulation of the basic principles of relativity are described in a way which anticipate Noll's papers in the subject; 
One may check by reading the biography of Noll [66] how close this list is to the list of subjects treated in his career by Noll. Noll's activity differs into two features: he did not study any bi-dimensional and one-dimensional continuum model and he simply ignored the existence of the variational principles or of the principle of virtual work.

Tartaglia's boasting statements questioned by Heiberg, Clagett and Dijksterhuis are paralleled by those in which Truesdell makes the apology of Noll. In Truesdell [103], one finds in a footnote on page 6 the following statement, which does not seem to have been widely accepted:

«The sixth of the problems Hilbert set for the twentieth century to solve was to formulate an axiomatic structure for physics, and especially for mechanics. Apart from a noteworthy attempt of Hamel in 1909, this problem was given scarcely any serious attention until it was taken up by Noll in 1957 . The content of Chapter I of this book derives essentially from the work of Noll and those who have accepted, applied, and extended his ideas.»

The reader will observe that Truesdell attributes in the aforementioned textbook to Noll all the axioms of mechanics and that Noll is cited therein at least 144 times, Lagrange 12 times in the body of the textbook and 10 times for critical comments in the introduction, Newton 36 times, Navier 8 times, Cauchy 171 times. Again Truesdell's biased historical vision is evident. His understanding of history of mechanics is also very partial. On page 6 of [103], one finds the following excerpt:

«In the words of Newton, ... Rational Mechanics will be the science of motions resulting from any forces whatsoever and of the forces required to produce any motions, accurately proposed and generated.»

Indeed, it is not Newton who first stated such a definition of mechanics. As in one of the previously excerpts, already Pappus made similar (and maybe more complete) statements by referring to Heron's school. Moreover, again in [103] on p. 63, one finds the following theorem:

«Theorem (Noll). The working of a system of forces is frame-indifferent if and only if that system and its associated system of torques are both balanced.»

This theorem is actually due to Gabrio Piola (see [26]). On p. 190 of [106] Truesdell's very partial interpretation of Piola's results is given.

\subsection{The positive role of erudite scholars whose work consists in "re-ordering" the house of knowledge}

Of course exactly as it happened with Commandino and Tartaglia, many positive effects of the publicistic activity by Truesdell and Noll can be found. First of all, they popularized European mechanics in the United States and made it accessible to the American academia and in particular to the scholars in engineering sciences. It is somehow ironic that the vision of Hellinger came back to Germany via its vulgarization by Truesdell as a mediator of Noll's works. However, this is what seems to happen and in the end it is not so strange if one thinks of how Archimedes' works managed to reach us. Moreover, Truesdell, Toupin and Noll helped to advertise the capabilities and potential of nonlinear continuum mechanics, which were clearly expounded in Hellinger's article but were ignored by the greatest part of scholars. This positive effects were also accompanied by the promotion of the so called Noll's Axioms. Axioms which one can find as true theorems in the postulation scheme presented by Hellinger who starts from different axioms.

Truesdell's work was useful in simplifying the extremely Bourbakistic formalism used by Noll, whose attitude towards mechanics can be regarded as exceedingly and not very efficiently formalistic. Truesdell made Noll's work more understandable and immediately useful to the engineering scientist. As Noll's conscious or unconscious source was Hellinger (or at least Hellinger's scientific milieu) ${ }^{13}$ this popularization work was definitively determinant in the advancement of engineering sciences.

As done by Tartaglia, also Truesdell used his position to promote himself and his pupils. While it is true that the majority of the mechanicians might never have heard of Piola if it had not been for Truesdell, it is also true that Truesdell promoted only the lesser achievements of Piola. Namely those conformed to his own mental scheme, which was forged by Noll. Remark that Toupin (who was influenced by reading Landau's textbooks) tried to direct Truesdell towards the true content of Hellinger's article. Nevertheless, he did not manage to succeed [personal communication at CanCNSM2013 Montreal 2013]. Acting in this way, Truesdell implied that Piola was much less than he really was, and so in this sense he can be accused of suppressing Piola. However, ironically, it is precisely this attempted suppression that inspired the present historical investigations. Such are the twists and turns (or Vico's and Polybius' cycles) of human history.

13 In Berlin Noll held a position as assistant of Prof. Istvan Szabò. Under Szabò's guidance Noll contributed largely to the writing of Szabo's textbooks "Mathematische Formeln und Tafeln" and "Integration und Reihenentwicklungen im Komplexen, Gewöhnliche und Partielle Differentialgleichungen" and "Technische Mechanik", cf. the exaggeratedly apologetic scientific biography "The Mathematical World of Walter Noll” by Y.A. Ignatieff [66]. Szabò was the typical chair director at a German university and he was a clever representative of the traditional German mechanics. 
The desired contribution of the present work is simply to revive the classical masters of mechanics, especially Hellinger and Piola, to prove how deeply indebted we are to these originators, and also how derivative and unoriginal some recent and contemporary mechanicians truly are. The intent is not to fix an uninteresting dispute of priority but to make to the mechanics community a service in tracing the origins of contemporary ideas to their true sources in order to make clear how original scientific progress was actually obtained in reality. This is needed at least for the education of new generations who should not believe into the heroic vision of the advancement of science. A community of scientists and not a single scientist is producing novel models and deep understanding of physical phenomena.

It seems reasonable to state that while Truesdell and Noll are definitely not the originators of any significant idea in mechanics, they did do much to "set the house in order" and to organize continuum mechanics into a palatable and penetrable subject, and to provide again a place for mechanics in the mathematics community - something that had been lacking due to its takeover by more applied scholars. Unfortunately, Truesdell and Noll seemed not to have any appreciation or understanding of the theory of structures, which mostly are one or two-dimensional "higher gradient continua", the development of which preceded modern "Cauchy" continuum mechanics. Remark that lower dimensional continua are completely ignored in the textbooks by Truesdell [105,106]. On the contrary, both Piola and Hellinger spent a lot of effort for including these lower dimensional continua in their conceptual scheme. Interestingly, Antman proclaiming himself as an equilibrated admirer of Truesdell (see Antman's preface to the third edition of [105]) uses intensively variational principles in his textbook [8] in order to deal with beams and rods. In Sect. 1 of Chap. 2 of the same book, Antman writes:

«We pay special attention to the Principle of Virtual Power and the equivalent Impulse-Momentum Law, which are physically and mathematically important generalizations of the governing equations of motion and which play essential roles in the treatments of initial and boundary conditions, jump conditions, variational formulations, and approximation methods. [...]

[...] A goal of this chapter is to show that it is easy to derive the equations correctly, much easier than following many modern expositions, which ask the reader to emulate the Red Queen by believing six impossible things before breakfast.

The correct derivation is simple because Euler made it so. Modern authors should be faulted not merely for doing poorly what Euler did well, but also for failing to copy from the master.»

It therefore seems that, while Truesdell did not remark that Euler based his deductions on variational principles (at least for the derivation of the elastica), Antman could recover successfully this method and effectively apply it. The historical reconstruction of this progress in science is sketched by Benvenuto in [11] on p. 212, referring to the letter from Daniel Bernoulli to Leonhard Euler from October the 20th, 1742. In this letter, Daniel Bernoulli asks Euler to solve the isoparametric problem of the elastica, i.e. to solve a variational problem. Two years later, Euler published the famous work "Methodus Inveniendi Lineas Curvas Maximi Minimive Proprietate Gaudentes" [44], where in the appendix I "De Curvis Elasticis", the elastica is treated. It would be interesting to investigate if Euler has been guided also by variational principles or by analogies to the elastica, when he came up with the principle of angular momentum. Then, as we know from Kirchhoff's kinetic analogy, the equations of the Kirchhoff beam (the three-dimensional inextensible beam whose cross sections remain rigid, plane and orthogonal with respect to the tangent vector of the centerline) being the three-dimensional analog to the elastica, are of the same form as the equations of a spinning top.

Truesdell has had always preferred very opinionated cultural positions. For example, he famously criticized, in a very sharp and hostile manner (cf. the introduction in [15]), the von Kármán plate theory, and ridiculed applied mathematicians for their theoretical interest in it. Much later, von Kármán was proved to be right in a paper by Friesecke et al. [50] based on Gamma-convergence, although the truth of his more formal reasoning was already self evident - once more here the Archimedean Mechanical Method is to be enlightened as a powerful heuristic tool. Another unfortunate circumstance concerns the Truesdellian attitude towards numerical computation. In the chapter "The Computer: Ruin of Science and Threat to Mankind" [104] he claims that science is ruined by the advent of powerful calculation tools. What happened in the subsequent years shows the myopia of his vision. It is somehow ironic that Truesdell's stimulus of nonlinear continuum mechanics could become effective and ready to technological applications not until the development of clever numerical codes, using powerful computers mostly based on variational principles. Luckily, Truesdellian authority, based on his capacities of vulgarization, did not manage to impeach the most beautiful applications of the ideas which he so greatly contributed to spread. This somehow schizophrenic attitude by Truesdell led him to be the advocate of mathematical rigor, but to refuse the methods of functional analysis leading to a correct formulation of variational principles and to be the champion of nonlinear theories but to refuse the only tool which has been conceived so far to solve effectively nonlinear problems.

Without being able to deepen this last point, we want to note that the essential (as distinct from optional) appreciation of variational methods, at least from the engineering perspective, may be due to Kirchhoff's early resolution of the boundary 
conditions in plate theory via a now-standard variational argument (see [67]), completing the profound program of mechanics and differential geometry initiated by Sophie Germain and of which also Gauss was a part. Kirchhoff's contribution seems to have established variational methods as a cornerstone of mechanics, and indeed the only rational and systematic procedure by which non-standard and even unexpected conditions could be understood. Actually, as proven by Piola's theorem, the elaborate "free-body" diagrams were later devised ad hoc only to provide ex post-facto justifications.

\section{Annotated translation of No. 9 (pp. 663-668)}

\section{B. Individualization for particular fields.}

9. Effective theory of elasticity. Now, it is about to exhibit, at which places in the general schemes developed in part $A$, the fundamentals for the treatment of the particular fields of the mechanics of continua, mainly used so far, are integrated; let us start with the theory of elasticity in the narrower sense, which has pointed this whole development in the right direction.

A purely elastic medium is characterized in this way, that the stress state inside [the medium] depends in each case merely on those expressions of the first derivatives of the deformation functions, which determine the pure shape change of the smallest parts with respect to the initial position:

$$
e_{a}=\frac{1}{2}\left(x_{a}^{2}+y_{a}^{2}+z_{a}^{2}-1\right), \quad g_{b c}=x_{b} x_{c}+y_{b} y_{c}+z_{b} z_{c} \quad(a, b, c)
$$

each of these quantities remain unchanged for orthogonal transformations of the $x$ - $y$-z-coordinate system being related to the deformed position, while they behave like the components of a symmetric dyad for transformations of the initial coordinates $a, b, c^{14}$. If one refers, as one usually does, to the case of the existence of a potential $\Phi$ of the most simple form of No. $7 a$, then the internal stresses are derived thus from the energy density function $\varphi$, which depends merely on the 6 deformation components $(1)^{15}$ :

$$
\varphi=\varphi\left(e_{a}, e_{b}, e_{c}, g_{b c}, g_{c a}, g_{a b}\right)
$$

thereby it is irrelevant, if one computes the density with respect to the volume element of the deformed or undeformed state, since the volume dilatation $\Delta$, appearing possibly as a factor, depends itself merely on the quantities (1).

In footnote 15, Hellinger follows the priority attributions dominated by the Anglo-Saxon school of mechanics. Since the second author having Italian as a mother tongue, we could also access the sources on this subject being available in Italian. Doubtlessly, it can be stated that Gabrio Piola did determine the correct strain measures directly for the case of finite deformations for $N$-th gradient continua. Furthermore, he also obtained the differential (local) form of the balance equations starting from the principle of virtual work followed by an integration by parts. In the case of a first gradient continuum, he found also the boundary conditions and introduced the Piola stress tensor in the reference configuration (called Piola's stress tensor in [106] and 2nd Piola-Kirchhoff tensor in [105]). Lastly, he introduced the Piola transformation from the reference to the actual configuration of the balance equations as well as of the boundary conditions for the first gradient continuum. It is not clear how in the Anglo-Saxon literature it could happen that the particular result concerning the transport of normals from one configuration to the other has been attributed to Nanson. The aforementioned series of works had been carried out by Piola in [80-83] between 1823 and 1850 - his last work has been published posthumous by Francesco Brioschi. Remark that the main results are included in a relatively primitive form already in the 1825 paper (submitted for publication before the 4th October 1824) which then are essentially completed in the work published in 1833. The other works include improvements and answers to the criticism raised by those (one can find always many) scholars who did not believe in the Lagrangian methods.

It seems therefore sure that the so-called Green strain measure and Nanson's Formula should be named after Piola, until eventually another antecedent author is found, who presented such definitions and results. It is astonishing that nobody in the very active Italian school of mechanics did actually read Piola's works, which although written in a very elegant Italian, with influences of Lombard dialect, are perfectly understandable also by contemporary Italian speakers having a minimum

\footnotetext{
14 Cf. IV 14, No. 17, 18, M. Abraham.

15 G. Green has first developed this ansatz for infinitesimal deformations (Trans. Cambr. Phil. Soc. 1838 = Math. Pap., London 1871, p. 248ff.); later (Trans. Cambr. Phil. Soc. 1839 = Math. Pap., p. 295ff.) he also stated [this ansatz] for finite deformations, but without carrying out the derivation of the equilibrium conditions. This has been done first by G. Kirchhoff (Sitzungsber. Wien, math.-phys. Kl. 9 (1852), p. 762), however, only with respect to isotropic bodies, and later in general by W. Thomson (Phil. Trans. Royal Soc. $153(1863)=$ Math. Phys. Pap., London 1910, vol. III, p. $386=$ Appendix C. to Vol. I, 2 of the Treat. on natur. philos. of Thomson and Tait).
} 
of literacy in their mother tongue. Possibly this is an effect of the cyclic behavior of Italian culture which is invariably xenophilous: since 1970 the preferred foreign scientists were considered those coming from the United States. For more details about the previous statements the reader is referred to [9,23,26].

From the formulas (4), (5) of No. 7 one extracts immediately the various expressions of the stress components, in particular [it] is ${ }^{16}$

$$
\left\{\begin{array}{l}
X_{x}=\sum_{(a b c)} \frac{\partial \bar{\varphi}}{\partial e_{a}} x_{a}^{2}+2 \sum_{(a b c)} \frac{\partial \bar{\varphi}}{\partial g_{a b}} x_{a} x_{b}+\bar{\varphi} \\
X_{y}=\sum_{(a b c)} \frac{\partial \bar{\varphi}}{\partial e_{a}} x_{a} y_{a}+2 \sum_{(a b c)} \frac{\partial \bar{\varphi}}{\partial g_{a b}}\left(x_{a} y_{b}+x_{b} y_{a}\right), \ldots \ldots,
\end{array}\right.
$$

and herefrom or from the remark, that the $e_{a}, \ldots$ do not change for every rigid rotation of the medium [and that] therefore also $\delta \Phi$ and consequently the virtual work of the stress dyad vanishes, the important relations follows

$$
X_{y}=Y_{x}, \quad Y_{z}=Z_{y}, \quad Z_{x}=X_{z} .
$$

What Hellinger mentions here is exactly the objectivity requirement for the internal energy which already has been postulated by Piola, cf. [26]. For further remarks on that issue, we refer to the first comment of Section 9 in this paper.

It is now the task of the particular theory of elasticity to study the form of the function (2) of the six variables for the individual media; meanwhile this general case of finite deformations recedes much in the theory of elasticity in favor of the infinitesimal deformations ${ }^{17}$. Here only the case of the isotropic elastic medium shall be emphasized especially; due to the equivalence of the directions in the medium, the 6 components of the shape change can enter (2) just by using their 3 orthogonal invariants with respect to transformations of the coordinate system $a, b, c$, i. e. it becomes

$$
\varphi=\varphi(A, B, C)
$$

where $A, B, C$ are the coefficients of the fundamental equation

$$
\left|\begin{array}{crr}
e_{a}-\Lambda, & \frac{1}{2} g_{a b}, & \frac{1}{2} g_{a c} \\
\frac{1}{2} g_{a b}, & e_{b}-\Lambda, & \frac{1}{2} g_{b c}, \\
\frac{1}{2} g_{a c}, & \frac{1}{2} g_{b c}, & e_{c}-\Lambda,
\end{array}\right| \equiv-\Lambda^{3}+A \Lambda^{2}-B \Lambda+C,
$$

which can be substituted certainly also by the square roots of this equation (lengths of axes of the deformation ellipsoid $)^{18}$. These formulas include readily the case, that the medium has "residual stresses" in the undeformed initial state; otherwise for vanishing e,g, the stress components (3) must vanish, i. e. the series expansion of $\varphi$ with respect to its six arguments must begin with quadratic terms ${ }^{19}$.

Furthermore, it has to be mentioned, that P. Duhem has applied his potential based approach (No. 7, (7)), which considers a direct effect of the states of deformation at two different points on each other, especially for isotropic elastic media. ${ }^{20}$ Thereby, the variables which enter $\varphi$ are besides the distance between the two considered points, the $2 \cdot 3$ invariants of the shape change [at those positions] as well as the characteristic quantities of the orientation of the deformation ellipsoids at both points with respect to each other and with respect to the connecting line segment.

Here, Hellinger attributes to Duhem the first formulation of what has been later called peridynamics. Therefore the fact that peridynamics could have been rediscovered in the 21 st century is even more astonishing, cf. [23, 26] for more details. Remark also how elegantly and simply (compared to the presentation given by Truesdell in Sect. 41 of [105]) the residual stresses are introduced by using a referential description.

\footnotetext{
16 J. Boussinesq, Mém. prés. par div. sav., Paris 20 (1872), p. 594; the authors cited in 15) have only expressions for the stress components with respect to the initial parameters $X_{a}, \ldots$ Cf. also Chap. III of the summarizing presentation of E. and F. Cosserat, Ann. de Toul. 10 (1896), p. J. 59.

$17 \varphi$ as a homogeneous quadratic function of the 6 arguments has been considered by W. Thomson, op. cit. ${ }^{15}$, p. 390, other forms, being characteristic for certain types of wave propagation, [have been considered] by J. Hamadard, Leçons sur la propagation des ondes (Paris 1903), p. 257ff.

18 Specific approaches for isotropic bodies can be found in G. Kirchhoff, op. cit. ${ }^{15}$, p. 773 and M. Brillouin, C. R. Paris 112 (1891), p. 1500.

19 Also already indicated for finite deformations by G. Green, op. cit. ${ }^{15}$, p. 298. Cf. also E. and F. Cosserat, op. cit. ${ }^{16}$, p. J. 70.

20 P. Duhem, Ann. Éc. Norm., (3) 21 (1904), p. $117 f f$.
} 
The biggest issue in the theory of elasticity is the consideration of infinitesimal deformations. The first terms of the components of the shape change (1) being linear in $\sigma$ are then, apart from the factor, in the former notations $\left(\right.$ No. 6, (6)): ${ }^{14}$

$$
\varepsilon_{a}=\frac{\partial u}{\partial a}, \quad \gamma_{b c}=\frac{\partial v}{\partial c}+\frac{\partial w}{\partial b} \quad\left(\begin{array}{c}
a, b, c \\
u, v, w
\end{array}\right)
$$

the function $\tilde{\varphi}$ however, from which according to No. 7a, (9) the stress components

$$
X_{a}=\frac{1}{\sigma} \frac{\partial \tilde{\varphi}}{\partial u_{a}}, \ldots
$$

emerge as linear functions of the displacement components, becomes

$$
\tilde{\varphi}=\sigma \varphi_{1}\left(\varepsilon_{a}, \gamma_{b c}\right)+\sigma^{2} \varphi_{1}\left(\frac{u_{a}^{2}+v_{a}^{2}+w_{a}^{2}}{2}, u_{b} u_{c}+v_{b} v_{c}+w_{b} w_{c}\right)+\sigma^{2} \varphi_{2}\left(\varepsilon_{a}, \gamma_{b c}\right),
$$

where $\varphi_{1}$ and $\varphi_{2}$ denote the linear and quadratic terms in the series expansion of $\varphi$ with respect to its 6 arguments, respectively, and [where] due to the sake of brevity throughout only one of each 3 arguments is written ${ }^{21}$.

If there appear no residual stresses, then $\varphi$ becomes a quadratic form of the 6 components of the infinitesimal shape change, and this is the case which provides the starting point of the ordinary theory of elasticity (cf. IV 24, No. 1, (1), O. Tedone); there it is then studied in particular of what forms this function will be, depending on the symmetry properties which the medium has with respect to the directions through a point[; is it] of the most general form with 21 constants (the elasticity constants) [or is it] up to the most special [form] with 2 constants (for the isotropic medium) (cf. IV 24, No. 2b, 2c). In this case the transformed variational principle (23) of No. $7 e$ is of particular simple form, as $H$ corresponds up to the sign with the energy density; its coefficients are the elasticity moduli of the medium.

Moreover, no complex (and eventually thermodynamical) argument is required for determining that the more general linear elastic material is described by 21 elastic coefficients. Hellinger derives the stress strain relationship starting from an energy. Hence, if the energy is quadratic then there are only 21 elastic coefficients, as the energy is determined by a symmetric $6 \times 6$ matrix. Many epigones of Truesdellism have studied elastic systems characterized by more than 21 elasticity coefficients, not being guided by any specific concept related to deformation energy.

Older than this line of thought is another perspective, which gives priority to the assumption, that all possible deformations of the medium shall be infinitesimal. The elastic medium appears here to be characterized in this way, that its potential $\varphi$ depends merely on the components of the shape change $\varepsilon, \gamma$ of the infinitesimal deformation: ${ }^{22}$

$$
\varphi=\varphi\left(\varepsilon_{a}, \varepsilon_{b}, \varepsilon_{c}, \gamma_{b c}, \gamma_{c a}, \gamma_{a b}\right)
$$

while the stress components emerge thereout as derivatives with respect to $u_{a}, \ldots$. In the most simple case of a medium without residual stresses, this makes certainly no difference, as far as one restricts oneself again to quadratic terms. However, one has also used this ansatz for the treatment of residual stresses ${ }^{23}$ and also for reaching an approximation of the natural processes going beyond Hooke's law by considering also terms of third and higher [polynomial] order ${ }^{24}$; naturally, these approaches apply for different media than for the former ones.

Starting from a slightly different point of view, J. Finger ${ }^{25}$ has tried to extend the basic formulas of the theory of elasticity for finite deformations; He not only considers the components of the shape change (1), but lets also depend $\varphi$ on all 9 derivatives $x_{a}, \ldots, z_{c}$, whereby he assumes - for an isotropic medium - merely symmetric appearance of the three coordinate directions as well as the existence of the relation ( $\left.3^{\prime}\right)$ and [whereby he] considers terms up to third order.

21 Such an expansion has already been used by G. Green, op. cit. ${ }^{15}$, p. 299. Cf. also H. Poincaré, Leçons sur la théorie de l'élasticité, Paris 1892, p. $47 \mathrm{ff}$. as well as E. and F. Cosserat, op. cit. ${ }^{16}$, p. J. $73 \mathrm{f}$.

22 This is the original ansatz by G. Green, op. cit. ${ }^{15}$, p. 249; cf. also IV 23, No. 5 b, Müller-Timpe.

23 Cf. e.g. H. von Helmholtz, Dynamik kontinuierlich verbreiteter Massen (Leipzig 1902), p. 93.

24 W. Voigt (Gött. Nachr., 1893, p. 534, math.-phys. Kl. 1894, p. 33; Ann. d. Phys, (3) 52 (1894), p. 536; Kompend. I, p. 339) uses for isotropic bodies also the orthogonal invariants of third order of $\varepsilon, \gamma$.

25 J. Finger, Sitzungsber. Wien $103^{\mathrm{II} a}$ (1894), p. 163, 231; see especially p. $175 \mathrm{ff}$. 
The reader will notice here once more that Hellinger denotes the coordinates of the reference placement by $(a, b, c)$, exactly as in Piola's works. Interestingly is the fact that after Piola at least Finger considered the study of second gradient elasticity to be of relevance. Once more one should investigate the erasure mechanisms which induced the loss of these results in a wide subsequent literature.

Also the theory of elasticity of bodies with one or two infinitesimal dimensions are subordinate to the potential-based approach (for two or one dimensions; No. 7c); compared to the three-dimensional elastic media, thereby the appearance of higher derivatives of the deformation functions in the energy density is new, as it is explained by the limit process of No. 8 a. This characteristics arises already in the expression of the potential

$$
\Phi=\int_{0}^{l} \varphi d a
$$

of the planar elastica, i. e. an elastic wire thought to be constraint to the plane $z=0$; namely, $\varphi$ becomes a function of the curvature radius $\varrho$ of the curve 26

$$
\varphi=\frac{E}{2} \cdot \frac{1}{\varrho^{2}}=\frac{E}{2}\left\{\left(\frac{d^{2} x}{d s^{2}}\right)^{2}+\left(\frac{d^{2} y}{d s^{2}}\right)^{2}\right\}
$$

provided that the inextensibility of the curve $(s=a)$ is added as a constraint to the requirement of the minimum of the potential - otherwise $\varphi$ is augmented by a term depending on the longitudinal dilatation $\frac{d s}{d a}$. Due to the limit process of No. $8 b$, one can relate the here appearing constants with the elasticity constants of the threedimensional extended medium.

For the spatial elastica the above mentioned (p. 659) fact is added to, that the displacement of the material of the wire with respect to the position of the center curve influences the energy. The detailed description is most conveniently done with the help of the Cosserat triad. One thinks of the orthogonal triad attached to every particle of the curve being oriented in the position of rest such that the third axis coincides with the tangent of the curve, while the other two indicate the border locations of the principal axes of the normal cut through the considered point for decreasing thickness of the wire; if one adds then additionally the constraint, that for every deformation the last axis of the triad is tangent to the curve:

$$
\alpha_{3}: \beta_{3}: \gamma_{3}=\frac{d x}{d s}: \frac{d y}{d s}: \frac{d z}{d s}=x_{a}: y_{a}: z_{a}
$$

which is of the form considered in No. 4c, then one has a Cosserat medium, which just describes the elastica. The property of elastic media, to have a potential depending only on the components of the shape change, corresponds here apparently with the assumption of a euclidean potential in the sense of the Cosserats (No. 7b, (17)), and since from (10) and No. 7, (16b) $\mathfrak{x}_{a}=\mathfrak{y}_{a}=0$ follows, the energy density can only depend on $\mathfrak{z} a$, which determines the elongation of the wire, and the components of the angular velocities $\mathfrak{p}_{a}, \mathfrak{q}_{a}, \mathfrak{r}_{a}$, which measure the geometric curvature of the deformed wire and the twist (Drall) of the material (cf. IV 25, No. 17, Tedone-Timpe) ${ }^{27}$.

$$
\varphi=\varphi\left(\mathfrak{z}_{a}, \mathfrak{p}_{a}, \mathfrak{q}_{a}, \mathfrak{r}_{a}\right)
$$

The special ansatz, which is provided by the theory of the elastica, is again the one of a quadratic form, and indeed - when as above additionally the inextensibility constraint is added -28 :

$$
\varphi=\frac{E}{2}\left(J_{1} \mathfrak{p}_{a}^{2}+J_{2} \mathfrak{q}_{a}^{2}\right)+\frac{C}{2} \mathfrak{r}_{a}^{2}
$$

hereby $E, J_{1}, J_{2}, C$ are material constants; If especially $J_{1}=J_{2}$ (what corresponds to a circular cross section of the wire), then the curvature $\frac{1}{\varrho^{2}}=\mathfrak{p}^{2}+\mathfrak{q}^{2}$ of the curve appears as in (9). By assumptions similar in kind about the relation between the position of the triad and the curve, one can represent all other types of bars, wires, strings treated in the theory of elasticity, how it has been developed extensively by E. and F. Cosserat ${ }^{29}$.

26 D. Bernoulli in a letter to Euler; P. H. Fuss, Cerresp. mathém. et phys., T. II, St. Pétersbourg 1843, p. 507. Cf. also L. Euler, Methodus inveniendi lineas maximi minimive proprietate gaudentes, Lausannae 1744, in the appendix "de curvis elasticis".

27 E. and F. Cosserat, Corps déformables, p. $37 \mathrm{ff}$.

28 Thomson-Tait, natural philos., new ed. I 2, p. $133 \mathrm{ff}$.; there also a more general quadratic ansatz is considered. Cf. also IV 25, No. 17.

29 E. and F. Cosserat, Corps déformables, No. 15-28. 
Very similar considerations apply to the theory of plates; which are treated here only briefly. One can consider a plate as a two-dimensional medium with oriented particles, whose triads shall stand [in such a way, that] the third axis stands always normal to the surface filled with the medium ${ }^{30}$; then $\mathfrak{z} a=\mathfrak{z}_{b}=0$ and

$$
\varphi=\varphi\left(\mathfrak{x}_{a}, \mathfrak{y}_{a}, \mathfrak{x}_{b}, \mathfrak{y}_{b} ; \mathfrak{p}_{a}, \ldots, \mathfrak{r}_{c}\right)
$$

depends on the stretch and the curvature of the deformed surface and the internal twisting of the matter on [the surface] in an orthogonal invariant manner. Also here, E. and F. Cosserat ${ }^{31}$ ) have carried out in detail, how one can derive thereout the energy theorem for the common approximation theory of the elastic plate ${ }^{32}$ as well as anyway for all types of elastic plates, membranes and shells ${ }^{33}$.

The purpose of an encyclopedia article to direct the interested reader to the relevant literature, re-directs also Hellinger to the work of the Cosserat brothers for what concerns the theory of beams and shells. Hellinger has a clear understanding of the fact that in these theories one intrinsically considers Cosserat continua.

\section{Annotated translation of No. 10 (pp. 668-670)}

10. Dynamics of ideal fluids. The ideal fluids are subordinate readily as a special case of those elastic media which allow for arbitrary finite deformations; they are characterized in this way, that only work must be expended for such deformations which are related to the volume dilatation or compression of the smallest particles ${ }^{34}$, and that the energy density $\varphi$ depends consequently only on the current volume dilatation measured by the Jacobian $\Delta$ at every point ${ }^{35}$ :

$$
\varphi=\varphi(\Delta)
$$

Since $\Delta$ is as an orthogonal invariant of the deformation, a function of the quantities $A, B, C(N o .9$, (4a)) $\left(\Delta^{2}=1+2 A+4 B+8 C\right)$, (1) is in fact only a special case of the ansatz (4) of No. 9. From No. 7, (5) the components of the internal stress related to this potential easily emerge as:

$$
\left\{\begin{array}{l}
X_{x}=Y_{y}=Z_{z}=\frac{d \varphi}{d \Delta}=p \\
X_{y}=Y_{x}=Y_{z}=Z_{y}=Z_{x}=X_{z}=0
\end{array}\right.
$$

i. e. the stress dyad determines a "fluid pressure" p acting uniformly in every direction. One obtains the same result also more directly ${ }^{36}$, when one determines with the help of the relation (cf. No. 2, $\left.\left(8^{\prime}\right)\right)$

$$
\delta \Delta=\Delta \cdot\left(\frac{\partial \delta x}{\partial x}+\frac{\partial \delta y}{\partial y}+\frac{\partial \delta z}{\partial z}\right)
$$

the variation of the total potential $\iiint \varphi d V_{0}$ and by equating [the variation] with the expression No. 3, (1) of the virtual work. - These considerations are valid both for hydrostatics and for hydrodynamics; Using (2) in the equations of No. $3 c$ and No. 5a, respectively, the well-known fundamental equations are obtained.

In hydrodynamics, equation (1) appears usually in a slightly different form. Since $\Delta$ is namely inversely proportional to the density $\varrho$ of the medium (No. 2, (7)), one can say, that [equation (1)] gives $\varphi$ as a function of $\varrho$, so that according to (2) also the pressure is given as a function of $\varrho$ :

$$
p=\frac{d \varphi}{d \Delta}=p(\varrho)
$$

the other way round, using (3), also the relation (1) is determined essentially. Usually, the "equation of state" of hydrodynamics is given in the form $(3)^{37}$.

\footnotetext{
30 Cf. E. and F. Cosserat, Corps déformables, p. $105 \mathrm{ff}$.

31 E. and F. Cosserat, Corps déformables, No. $41-46$.

32 Thomson-Tait, op. cit. $^{28}:$ I 2, p. $184 \mathrm{ff}$.

33 Cf. in particular the statement in IV 26, No. 5, H. Lamb.

34 Lagrange, Méc. anal., 1. part., sect. VIII, No. 1

35 J. Hadamard, Leçons sur la propagation des ondes (Paris 1903), p. $247 \mathrm{ff}$.

36 This is basically the procedure of Lagrange ${ }^{34}$; $c$. also sect. VII, No. 11.

37 Cf. the details in IV 15, No. 5, Love.
} 
As is generally known, the case of the incompressible fluid being characterized by the constraint

$$
\Delta=1
$$

looms large. For such a medium the equation of state (1) looses its meaning; however, if one approximates it according to No. $8 b$ as a "nearly incompressilbe" medium, the pressure $p=\frac{d \varphi}{d \Delta}$ becomes in the limit the Lagrangian multiplier of equation (4), if one adds [the equation] directly as a constraint to the principle of virtual work or to the principle of d'Alembert, in which then certainly only external and inertial forces and no more internal stresses enter ${ }^{38}$.

The content presented in this number until this point is essentially given in the works of Piola (see [9]). Piola also transforms the equilibrium equations which he obtains (also for fluids) in the reference configuration to their form in the actual configuration. However, Piola does not seem to be aware of the variational principle which Gauss (who was a contemporary to Piola) had developed and which is presented by Hellinger below. Remark that Duhem (see infra) did develop Piola's definition of nonlocal fluid and that Faye conceived the application of this generalized fluid for describing cometary trains.

The common presentations of hydrodynamics do not start with this perception of the fluid motion as a finite deformation subordinated to the theory of elasticity, but give priority to the so called Eulerian perception, i. e. the consideration of the velocity vector $x^{\prime}, y^{\prime}, z^{\prime}$ at every point. According to the equations (2), the fluid pressure is then directly defined between the stress components ${ }^{39}$ and the equations of motion are gained from the principle of d'Alembert or the principle of Gauss ${ }^{40}$

$$
\delta \iiint_{(V)} \frac{1}{2} \varrho \sum_{(x, y, z)}\left(x^{\prime \prime}-X\right)^{2} d V-\iiint_{(V)} p \sum_{(x, y, z)} \frac{\partial \delta x^{\prime \prime}}{\partial x} d V=0
$$

- for incompressibility p becomes a Lagrange multiplier.

Also especially for hydrodynamics $P$. Duhem ${ }^{41}$ has adapted his generalized potential-based approach No. 7 , (7), by letting the energy density $\varphi$ depend on the densities at both considered points and the distance between them; thereby he includes and generalizes forces, which H. A. E. Faye ${ }^{42}$ has taken into consideration for the explanation of the cometary train, in fact, attractive forces, whose intensity depend on the density of the acting particles.

\section{Annotated translation of No. 11 (pp. 670-674)}

11. Internal friction and elastic hysteresis. For moving elastic media and fluids there appear besides the so far discussed stresses and pressures also additional stresses, which are caused by internal friction, and therefore depend on the time derivatives of the deformation quantities ${ }^{43}$. If one uses the velocity components as functions of the actual position of every particle for the representation of the motion in the sense of Euler

$$
\mathfrak{u}=x^{\prime}=\mathfrak{u}(x, y, z ; t) \quad\left(\begin{array}{c}
x, y, z \\
\mathfrak{u}, \mathfrak{v}, \mathfrak{w}
\end{array}\right),
$$

then the 9 derivatives $x_{a}^{\prime}, \ldots, z_{c}^{\prime}$, which have been used above (No. 6, p. 640 und No. $7 f, p .657$ ) can be substituted by the 9 derivatives $\mathfrak{u}_{x}, \mathfrak{u}_{y}, \ldots, \mathfrak{w}_{z}$ which are linear functions of the former [derivatives]. The functions (1)

38 In Lagrange's presentation the incompressible fluid is the primitive; however, one cf. the remark in 124) (No. 8). (Note of the translators: This corresponds to the footnote 97 in [43], i.e. "Apparently, Lagrange had such a limit process in mind, as he denoted in his analytical mechanics the multiplier associated to $\omega=0$ as "force", which tries to change the function $\omega$; one shall compare in particular Sect. II, No. 9, Sect. IV, No. 6, 18, Sect. V, No. 53, Sect. VII, No. 21 of the first part, as well as the notes of J. Bertrand hereto - meanwhile the transition is not carried out in more detail. The presentation of the text is formulated following suggestions, which D. Hilbert has given in a "Göttinger Vorlesung" in the winter term 1906/7 for the treatment of incompressible fluids.»)

39 This corresponds to the perception of Euler; cf. IV 15, No. 2, 8, Love.

40 Cf. the extensive presentation of A. Brill, Mechanik raumerf. Massen ${ }^{64}$ ), p. $84 f f$. (Note of the translators: This corresponds to the footnote 25 in [42], i.e. «A. v. Brill, Vorlesungen zur Einführung in die Mechanik raumerfüllender Massen, Leipzig 1909.»)

41 P. Duhem, Ann. Éc. Norm. (3) 10 (1893), p. 183.

42 H. A. E. Faye, Paris C. R. 47 (1858), p. 939. 1043.

43 Cf. the historical details in No. 12 of IV 15, Love. 
determine the infinitesimal deformation, which the medium undergoes due to the motion during one time element; the components of the associated pure shape change (cf. No. 9, (5)) are:

$$
\mathfrak{e}_{x}=\frac{\partial \mathfrak{u}}{\partial x}, \quad \mathfrak{g}_{y z}=\frac{\partial \mathfrak{w}}{\partial y}+\frac{\partial \mathfrak{v}}{\partial z} \quad\left(\begin{array}{c}
x, y, z \\
\mathfrak{u}, \mathfrak{v}, \mathfrak{w}
\end{array}\right)
$$

and from these quantities the internal friction will merely depend on, as long as one considers analogously to the conditions in elastic media, that no frictional resistances can occur due to angular velocities of the particles ${ }^{44}$. By the way, one recognizes easily, that the components of the symmetric dyad determined by (2) with respect to the $a$-b-c-coordinate system just correspond to the time derivatives of the shape change components of No. 9, (1).

Here, Hellinger quickly discusses some relevant (and in 1913 well-known) kinematical relationships which allow for the representation of the time rate of deformation in terms of the Eulerian velocity gradient. In [103] several long elaborations are presented in this context.

So far, the theory of friction forces for finite deformations is developed completely only in hydrodynamics; the basic assumption thereby is the one of the existence of a dissipation function $D$, which is a homogeneous quadratic function of the quantities (2), and which moreover - according to the isotropic constitution of the fluid - depends merely on the orthogonal invariants ${ }^{45}$ :

$$
D=a_{1}\left(\mathfrak{e}_{x}+\mathfrak{e}_{y}+\mathfrak{e}_{z}\right)^{2}+a_{2}\left(\mathfrak{e}_{x}^{2}+\mathfrak{e}_{y}^{2}+\mathfrak{e}_{z}^{2}+\frac{1}{2}\left(\mathfrak{g}_{x y}^{2}+\mathfrak{g}_{y z}^{2}+\mathfrak{g}_{z x}^{2}\right)\right) .
$$

In accordance with No. $7 f,\left(29^{\prime}\right)$ and No. 3c, (8) the corresponding stress components with respect to the deformed state become

$$
\left\{\begin{array}{l}
X_{x}^{(1)}=\frac{1}{2} \sum_{(a b c)} \frac{\partial D}{\partial x_{a}^{\prime}} x_{a}=\frac{1}{2} \frac{\partial D}{\partial \mathfrak{u}_{x}}=a_{1}\left(\mathfrak{e}_{x}+\mathfrak{e}_{y}+\mathfrak{e}_{z}\right)+a_{2} \mathfrak{e}_{x} \\
X_{y}^{(1)}=\frac{1}{2} \sum_{(a b c)} \frac{\partial D}{\partial x_{a}^{\prime}} y_{a}=\frac{1}{2} \frac{\partial D}{\partial \mathfrak{u}_{y}}=\frac{1}{2} a_{2} \mathfrak{g}_{x y}, \ldots
\end{array}\right.
$$

Anyway, these stresses are added to the fluid pressure as influence of the internal friction; often one specifies the two constants in addition with the assumption that the arithmetic average of the three resulting normal pressures $p+X_{x}^{(1)}, p+Y_{y}^{(1)}, p+Z_{z}^{(1)}$ is equal to $p$, what results in $a_{1}=-{\frac{a_{2}}{3}}^{46}$.

In the effective theory of elasticity, so far, one has taken into consideration the internal friction only for infinitesimal deformations. In this case the quantities $\mathfrak{u}, \ldots ; \frac{\partial \mathfrak{u}}{\partial x}, \ldots ; \mathfrak{e}_{x}, \ldots ; \mathfrak{g}_{x y}, \ldots$ and $u^{\prime}, \ldots ; u_{a}^{\prime}, \ldots ; \varepsilon_{a}^{\prime}, \ldots$; $\gamma_{b c}^{\prime}, \ldots$ (in the notation of No. 6, (6) and No. 9, (5)) differ only by the factor $\sigma$, and accordingly the dissipation function becomes a quadratic [form and] the stress components [become] consequently linear forms of the time derivatives of the shape change components of the infinitesimal deformation. W. Voigt ${ }^{47}$ has thoroughly studied the dependences which can occur here.

Notice how naturally and without further elaboration Hellinger introduces here the dissipation function in both the finite and infinitesimal case. The sequence of reasons should be investigated for which for a long period and in some relatively numerous milieux it has been considered that dissipation phenomena could not be described in the framework of postulations based on variational principles. For a more elaborate discussion how to include dissipation phenomena in the variational framework, the reader is refferd to the paragraph following No. 7 of the second exegesis [42]. Hellinger also remarks that the representation formula for symmetric tensors depending isotropically on symmetric tensors can be applied for the constitutive equations for isotropic solids, when representing the Piola stress in terms of the (so-called) Green strain in the reference configuration or when representing the dissipative part of the Cauchy stress as a function of the rate of deformation in the spatial configuration.

In close relation to these approaches are the efforts to theoretically conceive the appearance of the elastic hysteresis in the context of the mechanics of continua, which however have not reached yet completely the large set of issues being treated here ${ }^{48}$. Typically is here in the first place the ansatz of L. Boltzmann ${ }^{49}$, which adds

\footnotetext{
44 G. G. Stokes, Cambr. Phil. Soc. Trans. 8 (1845) = Math. Phys. Papers I, p. 80.

45 W. Voigt, Kompendium I, p. 462 ff.; a more general Ansatz is given by P. Duhem Anm. Éc. Norm. (3) 21 (1904), p. $130 f f$.

46 Stokes, op. cit. ${ }^{156}$. Cf. also IV 15, Love, No. 12-14 and for the latter relation H. Lamb, Hydrodynamik (German edition Leipzig 1907$)$, § 314.

47 Abhandl. Ges. d. Wiss. Göttingen 36 (1889); Kompendium I, p. $456 f f ., 467 f f . ;$ Lehrbuch der Krystallphysik, Leipzig 1910, p. $792 f f$.

48 Cf. IV 31, No. 13 and 19 (v. Kármán).

49 Ann. d. Phys. u. Chem., Ergänzungsb. 7 (1876), p. 630.
} 
to the elastic stress components a time integral of the form as discussed in No. 6, (5); thereby he assumes what is certainly only valid for infinitesimal deformations - the integrand as linear function of the shape change components No. 9, (5) to be of similar form as the stress components are in the isotropic medium:

$$
\left\{\begin{array}{l}
X_{x}=\int_{-\infty}^{t}\left[a_{1}(t-\tau)\left\{\varepsilon_{a}(\tau)+\varepsilon_{b}(\tau)+\varepsilon_{c}(\tau)\right\}+2 a_{2}(t-\tau) \cdot \varepsilon_{a}(\tau)\right] d \tau \\
X_{y}=\int_{-\infty}^{t} a_{1}(t-\tau) \gamma_{a b}(\tau) d \tau
\end{array}\right.
$$

E. Wiechert ${ }^{50}$ has developed these formulas by special assumptions on the functions $a_{1}, a_{2}$ of $t-\tau$. A series of problems, belonging here, has been treated recently by V. Volterra ${ }^{51}$ (cf. p. 641).

The reader will remark here that a hint is given by the parallel reading of this part of Hellinger's article and the part in [105] dealing with materials with memory. The contributions by Boltzmann and Volterra are recognized more or less with exactly the same words. It is a reasonable conjecture that Truesdell and Noll used Hellinger as a source also in a conscious way. The reader shall recall that in Truesdell and Toupin [106], Hellinger is bitterly criticized, because of his choice to base the presently translated article on variational principles.

For the case of remaining shape changes, i. e. for plastic media, A. Haar and Th. v. Kármán ${ }^{52}$ have formulated the foundations concerning completely different aspects. They start with the variational principle No. 7, (23), in which (cf.p. 655) H corresponds for isotropic media to the energy density and becomes a homogeneous quadratic function of the first two orthogonal invariants of the (symmetric) stress dyad:

$$
2 H=a_{1}\left(X_{x}+Y_{y}+Z_{z}\right)^{2}+a_{2}\left(X_{y}^{2}+Y_{z}^{2}+Z_{x}^{2}-X_{x} Y_{y}-Y_{y} Z_{z}-Z_{z} X_{x}\right)
$$

To this variational problem with its three constraints (23a), No. 7, as characteristic property for plastic media, now the condition is added, that the largest shear stress appearing somewhere does not exceed a constant value $K$, i. e. that the differences between each of two roots of the equation

$$
\left|\begin{array}{ccc}
X_{x}-\Lambda, & X_{y}, & X_{z} \\
Y_{x}, & Y_{y}-\Lambda & Y_{z} \\
Z_{x}, & Z_{y} & Z_{z}-\Lambda
\end{array}\right|=0
$$

remain in absolute value below $K$ :

$$
\left|\Lambda_{1}-\Lambda_{2}\right| \leqq K, \quad\left|\Lambda_{2}-\Lambda_{3}\right| \leqq K, \quad\left|\Lambda_{1}-\Lambda_{3}\right| \leqq K .
$$

A solution of this variational problem with three equality and three inequality constraints will have various properties in various cases, depending on whether in the conditions (7) the equalities or inequalities hold. If all three inequalities hold, then one comes back to the common theory of elasticity, otherwise one arrives at the newly characteristic "semi-plastic" or "fully-plastic" states.

Basically, it would be a simple task to transfer this ansatz to sandy matter (theory of lateral earth pressure); in place of (7) other inequalities appear as constraints[. Inequalities] which express that the direction of the stress at every surface element lies not outside a certain "cone of friction". Meanwhile, there is missing here reliable information about the expression (6) of the deformation energy, such that this ansatz is so far only useful in the extreme case, where two of the inequality constraints hold as equality; then [this] results namely in differential equations which are independent of the special form of the energy expression ${ }^{53}$.

In the few sentences which conclude No. 11, Hellinger brilliantly resumes the methods used in plasticity or in the homogenized theory of granular media to find the equilibrium or evolution equations. The methods of constrained minimization are evoked and once more it is proven how fruitful variational methods are.

We want to highlight here the works by Francfort and Marigo [47] in which the methods described here by Hellinger show that the functional analytic framework of minimization of deformation energy in a (suitable) set of placement fields verifying eventually unilateral constraints is exactly what is needed also to develop predictive models for crack and/or damage growth.

50 Ann. d. Phys. (3) 50 (1893), p. 335.

51 Rom. Acc. Linc. Rend. (5) 18, 2 (1909), p. 295, 577; (19) 1 (1910), p. 107, 239; (22) 1 (1913), p. 529. Acta math. 35 (1912), p. 295.

52 Gött. Nachr., math.-phys. Kl., 1909, p. 212.

53 Haar and v. Kármán op. cit. ${ }^{52}$, p. 217. On the theory of lateral earth pressure cf. IV 27 (Reissner), besides the literature given there [cf.] also J. Sylvester Phil. Mag. (4) 20 (1860), p. 489 = Collected Papers, vol. 2, Cambridge 1908, p. 215 and J. Massau, Mémoire sur l'intégration graphique des équations aux dérivées partielles, fasc. 2 et 3. Mons 1902 and 1904. (Extrait des Annales des Ingénieurs sortis des Écoles spéciales de Gand.) 


\section{Annotated translation of No. 12 (pp. 674-675)}

12. Capillarity. The phenomena of capillarity include in contrast to the lastly considered phenomena insofar an essential new aspect, as they are related to the occurrence of interfaces of various media against each other. Hence, one will, as long as one holds on to the existence of a potential, derive the effects of capillarity from a potential constituent of the form (6) of No. 7a, namely from an integral over those interfaces:

$$
\Phi=\iint_{(S)} \bar{\psi} d S=\iint_{\left(S_{0}\right)} \psi d S_{0} .
$$

The ansatz for $\bar{\psi}$, which Gauss ${ }^{54}$ has derived by the above $\left.(p .647)^{93}\right)^{55}$ mentioned limit process, is, that $\psi$ depends not on the deformation functions but only on the constitution of the media adjacent to one another; then, when only homogeneous media occur, $\Phi$ is equal to a linear aggregate of the areas $S_{1}, S_{2}, \ldots$ of the various interfaces (in the deformed state $)^{56}$ :

$$
\Phi=C_{1} S_{1}+C_{2} S_{2}+\cdots
$$

The transformation of $\delta \Phi$ into the form No. $3 e$, (15) leads to the following efforts: a stress $C_{i} d s$ which appears only on the boundary curves of the surface patches $S_{i}$ [and which] acts within the surface $S_{i}$ orthogonally to every line element $d s$, and a pressure force, oriented normally to each internal surface element, [which is] up to the factor $2 C_{i}$ equal to its mean curvature. ${ }^{57}$

If one likes to relate the ansatz (1) closer to the usually prior perception of a spatially distributed energy, as it can be achieved by the computational transformation of the surface integral into a volume integral mentioned on $p$. 646, then one succeeds with the help of a limit process, which is similar to the one being used in No. 8 for related purposes. ${ }^{58}$ By restricting oneself for the sake of simplicity to a system with two media divided by a surface $S$, [media] which occupy the spatial parts $V_{1}, V_{2}$, one can substitute [the system] with one continuum representing the actual circumstances better[. One continuum] whose state changes continuously, but in the neighborhood of $S$ extraordinary fast and which includes as an abstract limit the system of the two media. The energy density $\varphi$ of such a medium ( $c f$. No. $7 a, p .645$ ) will depend also on the local derivatives of the deformation quantities, $i$. e. on the second derivatives of the functions $x(a, b, c), \ldots$; one will need to consider this dependence only in a small region $V^{(\varepsilon)}$ which surrounds $S$, while in the remaining domains $V_{1}^{(\varepsilon)}$ and $V_{2}^{(\varepsilon)}$ the consideration of the dependence on the deformation quantities of first order is enough. If one approximates now with such a described continuum the original system, by contracting $V^{(\varepsilon)}$ around $S$ indefinitely and simultaneously by letting $V_{1}^{(\varepsilon)}, V_{2}^{(\varepsilon)}$ converge to $V_{1}, V_{2}$, then by appropriately controlling $\varphi$ there will appear in the total potential besides the spatial potential $V_{1}$ and $V_{2}$ just a surface integral of the kind (1). If within $V^{(\varepsilon)}$, what from the ansatz. No. 10, (1) of hydrodynamics immediately suggests itself, one specially lets $\varphi$ depend on the derivatives $\frac{\partial \varrho}{\partial n}$ of the density normal to a system of parallel surfaces to $S$ occupying $V^{(\varepsilon)}$, by setting something like $\varphi=C \cdot \frac{\partial \varrho}{\partial n}$, then in the limit

$$
C \cdot \iint_{(S)}\left(\varrho_{1}-\varrho_{2}\right) d S
$$

is added to the potential, whereby $\varrho_{1}, \varrho_{2}$ are the boundary conditions of the densities in $V_{1}, V_{2}$ - this corresponds for constant densities just to the form (2). For the specific computation of this Ansatz, certainly there is again (cf. p. 660) the assumption, that the dependence of the equilibrium position of the approximated system on the parameter $\varepsilon$ is studied.

In the decade 1980-1990, a great interest on the subject presented in this number was shown by different groups. For what concerns the three-dimensional models of phase interfaces by means of generalized continua, we give here only a very biased list of research papers which exploited and developed the ideas presented by Hellinger. The reader will easily find

\footnotetext{
54 C. F. Gauss, Princ. generalia theoriae figurae fluiderum Comment. soc. reg. scient. Gotting. recent. $7(1830)=$ Werke V, p. 29.

55 This corresponds to the footnote 60 in [42], i.e. «E. g. in Navier's theory of the elastic potential (cf. IV 23, No. 5a, Müller-Timpe) and in the theory of capillarity of P. S. Laplace and C. Fr. Gauss (cf. V 9, No. 13, Minkowski)».

56 Op. cit. No. 18.

57 Cf. the extensive presentation of this derivation in V 9, No. $2 \mathrm{ff}$. (Minkowski).

58 For the following presentation cf. a note at the beginning of No. 5 in H. Minkowskis paper V 9; the same procedure has been followed by D. Hilbert in a Göttinger Vorlesung in the winter term 1906/07.
} 
from them the research streams to which we refer, [2-5, 12, 13, 24, 25,30,31,51-53,74,91,94,95]. Using different methods all the previous papers create also a "limit" model which applies when the thickness of the interface is small.

The concepts formulated by Hellinger were more or less deeply (and more or less rigorously) explored in the subsequently referred literature also for what concerns the so-called "direct" bi-dimensional continuum models for phase interfaces. These models were introduced to generalize the treatment first proposed by Laplace and Young (see e.g. [32-34,68] and references cited therein).

The very same ideas have been rediscovered subsequently, more or less independently for instance in [6,48,61]. When comparing these last papers with those already available in the literature, one may conclude that the present well-known models belong all to the class carefully described by Hellinger, with some nominalistic improvements and some novel applications. The nomenclature "sharp interface" is suggestive and most likely is one of the main contributions which these papers add to the existing literature for what concerns the model formulation. On the other hand the applications presented therein are of some interest.

\section{Annotated translation of No. 13 (pp. 675-679)}

13. Optics. In order to introduce the optical phenomena within the scheme of the general mechanics of continua, one considers as is well known the components $u, v, w$ of the light vector as displacement components of particles of a deformable space-occupying medium (light ether); it is enough for the purposes of optics to restrict oneself thereby to infinitesimal deformations. ${ }^{59}$ For this perception it is not at all necessary, to attribute to the light ether - as in the effective elastic theory of light - the property of an elastic medium in the narrower sense; on the contrary one obtains the correct formulas of optics just then in the most simple way, when one does not attribute to the components of the pure shape change $($ No. 9, (5)) but to the one of the rotation of the volume elements

$$
\frac{1}{2} \xi=\frac{1}{2}\left(\frac{\partial w}{\partial b}-\frac{\partial v}{\partial c}\right), \quad \frac{1}{2} \eta=\frac{1}{2}\left(\frac{\partial u}{\partial c}-\frac{\partial w}{\partial a}\right), \quad \frac{1}{2} \zeta=\frac{1}{2}\left(\frac{\partial v}{\partial a}-\frac{\partial u}{\partial b}\right)
$$

the determining role for the value of the deformation energy. This idea originates from J. Mac Cullagh ${ }^{60}$, and he succeeded in this manner not only to achieve the differential equations, but also - beyond the elastic theory of light - [to achieve] the correct boundary conditions of optics.

For isotropic transparent media Mac Cullagh's Ansatz lies therein to assume an energy density proportional to the squares of the absolute value of the rotation vector (1) within the light ether ${ }^{61}$ :

$$
\varphi=\frac{1}{2} A\left(\xi^{2}+\eta^{2}+\zeta^{2}\right)=\frac{1}{2} A \sum_{\substack{a b c \\ u v w}}\left(w_{b}-v_{c}\right)^{2} .
$$

Then it follows from No. 9, (6) for the stress components

$$
\begin{gathered}
X_{a}=Y_{b}=Z_{c}=0 \\
Z_{b}=-Y_{c}=A \xi, X_{c}=-Z_{a}=A \eta, Y_{a}=-X_{b}=A \zeta
\end{gathered}
$$

and the equations No. 5, (2) for the motion reads therefore as

$$
\varrho u^{\prime \prime}=A\left(\frac{\partial \eta}{\partial c}-\frac{\partial \zeta}{\partial b}\right)=A\left(\Delta u-\frac{\partial\left(u_{a}+v_{b}+w_{c}\right)}{\partial a}\right) \quad\left(\begin{array}{c}
u, v, w \\
a, b, c \\
\xi, \eta, \zeta
\end{array}\right) ;
$$

which are, when one adds the condition $u_{a}+v_{b}+w_{c}=0$ of the incompressibility, precisely the oscillation equations of optics. Likewise, the boundary conditions of optics are included in the boundary conditions, which are obtained similarly to No. 3c, (5b) and which express for instance for the interface between two media with different constants $A$, that both generated expressions

$$
A(\eta \cos n c-\zeta \cos n b) \quad\left(\begin{array}{c}
\xi, \eta, \zeta \\
a, b, c
\end{array}\right)
$$

59 In what follows, we refrain for the sake of brevity from adding the infinitesimal factor $\sigma$.

60 Mac Cullagh, An essay towards a dynam. theory of cryst. reflexion and refraction, Trans. Roy. Irish Acad., 21 (1839) = Coll. Works (Dublin 1880), p. 145. - Cf. also V 21, No. 24 (Wangerin) and V 22, No. 1 (W. Wien).

61 Cf. also the presentation of W. Voigt, Kompendium II, p. 563. 
coincide.

The integral of the energy density (2) allows for a transformation, by virtue of which [the result] coincides up to a boundary integral with the volume integral of

$$
\frac{1}{2} A \sum_{\substack{u v w \\ a b c}}\left\{\left(w_{b}+v_{c}\right)^{2}-4 v_{b} w_{c}\right\}
$$

but this is the energy density of a purely elastic isotropic medium, whose Lamé parameters $\lambda, \mu$ are related according to $\lambda=-2 \mu=-2 A$. W. Thomson (Lord Kelvin) has used a medium of just this constitution for the explanation of optical phenomena ${ }^{62}$

Here Hellinger shows, with a historical example, the power of the principle of virtual work or its weaker form, i.e. the principle of least action. One could start from "the balance equations" (3a), postulating them as any Truesdellian would like to do. Maybe they could even persuade themselves that this balance equation is "physically" well grounded, with some exoteric reasoning. Remark that here we use the word exoteric in its original meaning. ${ }^{63}$ However, the terrible problem to be faced then is to find the boundary conditions which are suitable to complete the differential (local) form of postulated balance equations into a well-posed problem. Everybody familiar with the calculus of variations knows that this problem can be very difficult and that there are, in general, infinitely many boundary conditions which can be chosen, and that there are also infinitely many boundary conditions which lead to ill-posed problems. The "Deus ex Machina" which is invoked too often is the mysterious "physical sense", whatever this expression may mean. Of course, if one knows the right results because one had read it in a book (where possibly the principle of virtual work and a suitable integration by parts has been used) then the physical sense is greatly enhanced! The fundamental idea of the mechanicism of the 19th century was that the light is a perturbation of a mechanical continuum, called "luminiferous ether". Now, Lamé and Lord Kelvin assumed that ether was a linear elastic deformable solid whose deformation energy is given by the previous unlabeled formula. They integrated by parts and found the boundary conditions consequent with such an assumption. Indeed variational principles lead the scholar to make consistent assumptions for differential equilibrium equations and boundary equilibrium conditions. However, the obtained boundary conditions led to predictions which are not consistent with the experimental evidence, while the deformation energy postulated by Mac Cullagh DID produce a perfect agreement with experiments. This was an important step ahead in science. The luminiferous ether disappeared out of the mind of the scholars, being a mechanical model surely not suitable to describe phenomenology. However, the equations invented to describe its behavior (developed by assuming its imaginary existence) did remain in every physics book since then, because of their predictive value. The whole physics had to be changed to give a coherent meaning to these equations.

Again: physical interpretations are flowing away while Lagrangians stay much more stably in the physics textbooks. As remarked (see below) by the Cosserat brothers the ether is a Cosserat medium whose energy depends on the "absolute" rotation with respect to the absolute Euclidean space.

Mac Cullagh has carried out his ansatz in particular for the optics of crystalline media, by setting $\varphi$ equal to a quadratic form of $\xi, \eta, \zeta$ (with constant coefficients) ${ }^{64}$ :

$$
2 \varphi=A_{11} \xi^{2}+2 A_{12} \xi \eta+\cdots+2 A_{23} \eta \zeta+A_{33} \zeta^{2}
$$

Completely analogous as above the differential equations follow as

$$
\varrho u^{\prime \prime}=\frac{\partial \mathrm{H}}{\partial c}-\frac{\partial \mathrm{Z}}{\partial b}, \text { where } \Xi=\frac{\partial \varphi}{\partial \xi} \quad\left(\begin{array}{l}
\Xi, \mathrm{H}, \mathrm{Z} \\
u, v, w
\end{array} ; \begin{array}{c}
\xi, \eta, \zeta \\
a, b, c
\end{array}\right)
$$

while in the boundary conditions the [subsequent] expressions appear:

$$
\mathrm{H} \cos n c-\mathrm{Z} \cos n b . \quad\left(\begin{array}{c}
\Xi, \mathrm{H}, \mathrm{Z} \\
a, b, c
\end{array}\right)
$$

62 W. Thomson, Phil. Mag. (5) 26 (1888), p. 414ff. Cf. also V 21, No. 31 (Wangerin).

63 «[Aristotle] In the mornings he gave lectures to a narrow circle, in a strictly formal and scientific way, upon the higher branches of science; while in the afternoons he conducted conversations upon more popular themes with a less select audience. The former were called his esoteric, the latter his exoteric, discourses.» Excerpt from Davidson [22].

64 Cf. Mac Cullagh, works ${ }^{60}$, p. 156, where (4) readily emerges as a sum of squares. See also the presentation in P. Volkmann, Vorles. über die Theorie des Lichtes, Leipzig 1891, p. 260, 294. 
E. und F. Cosserat have indicated ${ }^{65}$, that their "euclidean potential" includes also these fundamental approaches of Mac Cullagh.

Based on this foundation, one can try, with an enhancement of the potential-based approach according to the direction discussed in No. 7, to include all equations required for the various optical problems; in this way W. Voigt proceeded systematically in his Kompendium. ${ }^{66}$

In the first place, he gains the transition to the dependence of the optical appearance of the color (oscillation duration $\tau$ ), by adding to the terms of (4) equally generated quadratic forms of the time derivatives $\xi^{\prime}, \eta^{\prime}, \zeta^{\prime}$ or $\xi^{\prime \prime}, \eta^{\prime \prime}, \zeta^{\prime \prime}$ and so on, certainly with the simultaneous restriction that the light vector realizes throughout sine oscillations with period $\tau$. Now he uses Hamilton's principle in the form No. 7, (25), and by integration by parts with respect to time he can transform this additional terms such ${ }^{67}$, that eventually again a quadratic form of $\xi, \eta, \zeta$ just like (4) acquires the position of the energy density, save that their coefficients $A$ are now functions of $\tau$; The type of these functions depend on the medium and determine its behavior with respect to different colors.

In a similar way Voigt uses also quadratic terms formed with time derivatives of different orders of $\xi, \eta, \zeta$ and shows, that one can reduce them to one essentially new characteristic constituent of the energy density:

$$
B_{1}\left(\zeta^{\prime} \eta-\eta^{\prime} \zeta\right)+B_{2}\left(\xi^{\prime} \zeta-\zeta^{\prime} \xi\right)+B_{3}\left(\eta^{\prime} \xi-\xi^{\prime} \eta\right)
$$

thereby $B_{1}, B_{2}, B_{3}$ are given constants or functions of $\tau .{ }^{68}$ The additional terms, which appear consequently in the differential equations and the boundary conditions, are extracted easily from the general formulas; they describe the change, which the motion of light undergoes under [the influence] of a magnetic field (magnetic activity), and indeed, the quantities $B$, which transform like the components of an axial vector, depend on the position of the magnetic axis at the considered position and the magnetic field strength. ${ }^{69}$

In the third place, Voigt considers finally also aggregates of products between a first time derivative of $u, v, w$ and one of $\xi, \eta, \zeta$. Also they have, which is shown by similar transformations ${ }^{70}$, as a result the appearance of more simple terms in the expression of the virtual work, for which it is typical [that]

$$
C(u \delta \xi+v \delta \eta+w \delta \zeta)
$$

The differential equations hereto are easily obtained; they provide the phenomena of the naturally active media. ${ }^{71}$

Mac Cullagh himself has considered these media in the same way, by giving the energy density an additional term containing derivatives of second order

$$
\frac{1}{2} C \sum_{\substack{u v w \\ \xi \eta \zeta \zeta}} \xi\left(\frac{\partial^{2} u}{\partial a^{2}}+\frac{\partial^{2} u}{\partial b^{2}}+\frac{\partial^{2} u}{\partial c^{2}}\right)
$$

Then the differential equations obtain as additional terms derivatives of third instead of first order as in Voigt. ${ }^{72}$

Voigt gains the transition to absorbing media, by using a dissipation function (No. 7f, p. 657, No. 11, p. 671) given as a quadratic form of derivatives $\xi^{\prime}, \eta^{\prime}, \zeta^{\prime} 73$

$$
2 D=m_{11} \xi^{\prime 2}+2 m_{12} \xi^{\prime} \eta^{\prime}+\cdots+m_{33} \xi^{\prime 2} m_{33} \zeta^{\prime 2}
$$

[and] adding to the virtual work

$$
-\left(\frac{\partial D}{\partial \xi^{\prime}} \delta \xi+\frac{\partial D}{\partial \eta^{\prime}} \delta \eta+\frac{\partial D}{\partial \zeta^{\prime}} \delta \zeta\right)
$$

this leads simply to the addition of the complexes $\frac{\partial D}{\partial \xi^{\prime}}, \ldots$ to the $\Xi, \ldots$ in the formulas $(4 a),(4 b){ }^{74}$

65 E. und F. Cosserat, Corps déform., p. 151.

66 See particularly V. Teil (Optik), $\$ 7$ (Bd. II, p. $563 f f$.$) as well as Kap. II, III of this part and cf. also II. Teil, § 34$ (Band I, p. $486 f f$.$) , where the force$ effects are formulated directly without the communication of a potential.

67 Op. cit. $^{66}$, p. 569.

68 op. cit. p. $568 f f$.

69 op. cit. p. 572, 679 ff.

70 op. cit. $572 f f$.

71 Op. cit. p. 574, 687ff.

72 Mac Cullagh, Proc. R. Irish Ac. II (1841), p. 96 = Works, p. 187. Cf. also P. Volkmann, Theorie des Lichtes, p. 414 ff.

73 Corrections of the last term in the following equation are made by the translators.

74 op. cit.p. 575 f., $708 f f$. 
While all this considerations concern the expression of the potential energy, one can likewise try to use in optics generalizations of the most simple expression $\frac{1}{2} \varrho\left(u^{\prime 2}+v^{\prime 2}+w^{\prime 2}\right)$ of the kinetic energy, as they are discussed in No. 5 d. In this direction lies the already mentioned ansatz of J. W. Strutt (Lord Rayleigh) ${ }^{75}$, to assume the kinetic energy per unit volume of the light ether as a general quadratic form of the velocities $u^{\prime}, v^{\prime}, w^{\prime}$; thereby linear combinations of accelerations $u^{\prime \prime}, v^{\prime \prime}, w^{\prime \prime}$ do appear on the left hand side of the optical equations.

Here a general comment is needed: one has to admit, looking at the evolution of the field theories presented here, that the guidance given by Hamiltonian or Lagrangian methods has proven to be the most effective in the elaboration of novel theories - or in other words - when a scholar does not have the guidance of sources which suggest how to develop a theory. Such a lucky circumstance surely guided Galileo and Newton, who admittedly said "If I have seen further, it is by standing on the shoulders of giants" probably rephrasing previous authors, among whom we can list Bernard of Chartres. We claim that one can be guided by "physical sense" only if one has an "a priori" knowledge of how the theory should be developed. In absence of such an a priori knowledge, one can only start to model conservative systems by postulating more and more complex Lagrangians and checking which are the possibilities which they allow for. Once a satisfactory description of conservative phenomena is developed, one may add for instance dissipation potentials to include nonconservative ones.

\section{Annotated translation of No. 14 (pp. 679-681)}

14. Relations to electrodynamics. The fundamental equations of electrodynamics are in their form, as it is well known, essentially included in the optical fundamental equations and thereby [included] in the general scheme of the mechanics of continua. To speak only of the isotropic medium, if one interprets namely the time derivatives of the components $u, v, w$ of the just considered light vector up to a constant factor as vector of the electric field strength $\mathfrak{E}$ :

$$
u^{\prime}=\gamma_{1} \mathfrak{E}_{a}, \quad v^{\prime}=\gamma_{1} \mathfrak{E}_{b}, \quad w^{\prime}=\gamma_{1} \mathfrak{E}_{c},
$$

and likewise the components of the rotation of a volume element as components of the magnetic field strength $\mathfrak{H}$ :

$$
\xi=\gamma_{2} \mathfrak{H}_{a}, \quad \eta=\gamma_{2} \mathfrak{H}_{b}, \quad \zeta=\gamma_{2} \mathfrak{H}_{c}
$$

then for a suitable choice of the constants $\gamma_{1}, \gamma_{2}, A, \varrho$ the equations (3a) and (1) of No. 13 transform to

$$
\left\{\begin{aligned}
\frac{\partial \mathfrak{H}_{c}}{\partial b}-\frac{\partial \mathfrak{H}_{b}}{\partial c}=\frac{\varepsilon}{c} \mathfrak{E}_{a}^{\prime} & & (a, b, c), \\
\frac{\partial \mathfrak{E}_{c}}{\partial b}-\frac{\partial \mathfrak{E}_{b}}{\partial c}=-\frac{\mu}{c} \mathfrak{H}_{a}^{\prime} & & (a, b, c),
\end{aligned}\right.
$$

and these are just Maxwell's Fundamental Equations in the free ether. ${ }^{76}$ A further term corresponding to external forces that can be additionally included in equations ( $3 a)$ finds its interpretation as electric current in the first three equations of (1). Similarly one can also obtain the electromagnetic equations for anisotropic media.

The sentences which one finds here, show how deeply Hellinger has understood not only the structure of field theories and their relationship with physical phenomena, but also the historical development of such theories. Here, he refers about what is sometimes called (see Russo [93]) "a fossil nomenclature". Even nowadays, when the attitude towards electromagnetism is the one described in the following lines, in any textbook one reads about so-called "Electric Displacement" vector field. While talking with many experts in electromagnetism the second author was astonished to discover that the great majority of them had NEVER tried to understand why such a vector should be named "displacement". Even more, they never had asked themselves about this strange circumstance. Why displacement? It is clearly said by Hellinger that this is a reminiscent of the "ancient" mechanistic concept of ether. The mechanistic ideas have been removed, but the name had been frozen in the literature and nobody dares to change it.

In the presentations, in which the general fundamentals of electrodynamics are found, however, usually one does not start with the perception to relate the electric and magnetic quantities with the displacements of a medium in such a direct way; one considers these quantities rather as "physical parameters" in the sense of No. $2 b$, from which, if necessary, one interprets some as dependent on the motion of an immaterial medium - of the electricity. In addition also the motion of the material medium, in which the process takes place, can be taken

75 J. W. Strutt, Phil Mag. (4) 41-43 (1871, 1872). Cf. also V 21, No. 29 (Wangerin).

76 Cf. W. Thomson, Math. phys. pap. 3 (London 1890), p. 436ff. One can also interchange the role of the electric and magnetic field strength; cf. about the various possible interpretations V 13, No. 42, H. A. Lorentz. 
into consideration. The equations of electrodynamics now relate all these quantities directly with forces, stresses, energy densities. The variational principles, into which one has often condensed them according to the approach of H. A. Lorentz ${ }^{77}$ and H. v. Helmholtz ${ }^{78}$, are then in a certain sense analogous to the mechanical one, save that they are very much more complicated due to the bigger amount of involved quantities. One shall compare the papers of H. A. Lorentz on these problems of special electrodynamics, in particular V 13, No. 35-39 and V 14, No. 8, 9. Only a special case shall be highlighted in addition, in being typical how such physical parameters can be treated in the former sense within the constitutive laws of a material medium.

Electrodynamics of deformable media is presented shortly here, and the references to relevant literature (available in 1913) is given. A careful investigation of this available theory at the period of Hellinger most likely will allow the assessment of the true originality of the contents of more modern textbooks (see e.g. $[41,69,73]$ )

Let an electric field $\mathfrak{E}$ be excited in an elastic medium; the generalization of the former ansatz is then the one, that the energy density $\varphi$ depends apart from the deformation quantities $e_{a}, \ldots, g_{a b}, \ldots$ also on the components of the field strength ${ }^{79}$ :

$$
\varphi=\varphi\left(e_{a}, \ldots, g_{a b}, \ldots ; \mathfrak{E}_{a}, \mathfrak{E}_{b}, \mathfrak{E}_{c}\right) .
$$

Using the former formulas No. 7, (4), the stress components appear unchanged, which depend consequently also on the electric field strength; Apart from that, the potential has to be minimized also with respect to the variation of the field strength $\mathfrak{E}$, and thereof the equations for the "electric torques" are obtained

$$
\mathfrak{P}_{a}=\frac{\partial \varphi}{\partial \mathfrak{E}_{a}}, \quad \mathfrak{P}_{b}=\frac{\partial \varphi}{\partial \mathfrak{E}_{b}}, \quad \mathfrak{P}_{c}=\frac{\partial \varphi}{\partial \mathfrak{E}_{c}},
$$

which show a dependence of the electric state on the deformation. In both systems of formulas or in the consequently following relation of the kind

$$
\frac{\partial X_{a}}{\partial \mathfrak{E}_{a}}=\frac{\partial \mathfrak{P}_{a}}{\partial x_{a}}
$$

the so-called reciprocity theorems ${ }^{80}$ are included, which play a crucial role in all these phenomena which relate various fields; if a change of one physical parameter causes the change of another related stress component, then also a variation of this parameter causes a certain change of the stress component related to the first. In these formulas the phenomena of piezoelectricity are included, which have been studied thoroughly with the help of simple forms of $\varphi$ according to the symmetry conditions of crystalline media. ${ }^{81}$

Once more the power of variational methods is underlined by Hellinger, who manages to clarify how naturally in such a conceptual framework the reciprocity theorems are found and how naturally one can understand piezoelectricity.

\section{Annotated translation of No. 15 (pp. 682-685)}

15. Introduction of the thermodynamical foundations. From the so far developed basic formulas of the mechanics of continua, there are two ways to climb up to the more comprehensive foundations of thermodynamics, which, within the scope of this article, are outlined only in a nutshell. One [way] builds on the equations of kinetics, e.g. Hamilton's principle of the generalized form No. 7, (26), and relies on the assumption that some relation $\omega$ of the motion and the spatial derivatives thereof does not appear explicitly in the integrand $\varphi$, but rather its time derivative. One can treat such a "hidden coordinate", which one can see instead of the motion

77 H. A. Lorentz, La théorie électromagn. de Maxwell (Leiden 1892), § 55ff.)

78 H. v. Helmholtz, Das Prinzip der kleinsten Wirkung in der Elektrodynamik. Ann. d. Phys. 47 (1892) p. 1 = Wissensch. Abh. III (Leipzig 1895), p. 476.

79 After W. Voigt had treated first the theory based on the directly formulated dependence of the stress and torque components on the deformation and field strength (Abhandl. Ges. d. Wiss. Göttingen, 36, 1890), P. Duhem, Leçons sur l'électricité 2 (1892), p. 467, E. Riecke (Nachr. Ges. d. Wiss. Göttingen 1893, p. 19) and W. Voigt (ibid., math.-phys. Kl. 1894, p. 343) have used the potential-based approach; For more details see V 16, No. 8, F. Pockels.

${ }^{80}$ Cf. hereto Voigts Kompendium II, p. 106. - One can transmit these reciprocity theorems, which are mostly treated for the case of finitely many degrees of freedom (cf. J. J. Thomson, Anwendungen der Dynamik auf Physik und Chemie [Leipzig 1890] and H. von Helmholtz, Journ. f. Math. 100 (1887), p. 137 = Wiss. Abh. III, p. 203ff.) in the wider extent to continua.

81 Cf. the extensive presentation in V 16, No. 8-10, F. Pockels. 
as motion determining, just as one does it in Helmholtz's theory ${ }^{82}$ of cyclic systems in the mechanics with finitely many degrees of freedom: With the help of elimination methods of the calculus of variations which are used in the theory of canonical transformations of dynamics ${ }^{83}$, one introduces in the variational principle instead of $\omega^{\prime}$ the derivative $\pi=\frac{\partial \varphi}{\partial \omega^{\prime}}$ and obtains consequently for the limit case of a state change, for which the velocities and accelerations of the remaining coordinates (except $\omega$ ) are indefinitely small, a variational principle which differ from the principle of virtual displacements only by the additional term $\omega^{\prime} \cdot \delta \pi$. The volume integral of this term has then the interpretation of the added heat quantity for a virtual displacement, while $\omega^{\prime}$ and $\pi$ represent temperature and entropy[, respectively]. Similar considerations are always treated extensively within the thermodynamics of systems with finitely many degrees of freedom ${ }^{84}$; but after all, an explicit application within the mechanics of continua seems not to be available.

The presentation of the first method presented by Hellinger in the previous sentences is rather concise. We will postpone further comments and investigations about it in a subsequent paper. However, it is evident that here it is reported about a stream of researches which has been considered sometimes in the subsequent literature and actually started some relevant investigations in thermodynamics. Actually, the effort consists in defining the purely thermal concept of temperature (or equivalently entropy) in terms of mechanical quantities once mechanical evolution has been subjected to a kind of "irreversibility" constraint. The papers [45] and [71]) are among the few which are investigating such a possibility. They stem from the basic ideas by von Mayer and Joule about the transformation of mechanical work into thermal energy and vice versa and take into account the phenomenological limitations observed in this transformation.

The second way is of much more formal nature and builds directly on the formal understanding being expressed so far. To the deformation functions - we restrict us for the sake of simplicity to statics - a "physical parameter" in the sense of No. $2 b$

$$
s=s(a, b, c)
$$

is added, whose value at every point describes the "thermal state" of the medium; one denotes it as entropy of the medium at this point, evaluated per unit mass, by calling the integral

$$
\iiint_{\left(V_{0}\right)} s(a, b, c) \varrho_{0} d V_{0}=\iiint_{V} s(x, y, z) \varrho d V
$$

entropy of a portion $V_{0}$ of the medium. For a virtual displacement of the continuum, also $s$ will undergo an infinitesimal virtual change $\delta s$. According to the second law of thermodynamics, one can then enhance the principle of virtual displacements in the following way ${ }^{85}$ :

Additionally to the virtual work $\delta A$ of all force contributions, equitably the "heat supply" appears for a virtual displacement:

$$
\delta Q=\iiint_{(V)} \Theta \delta s \varrho d V
$$

thereby "temperature" $\Theta$ denotes a factor being similar to the stress components[. A factor], which is given for every medium in its characteristic way in its dependence on the deformation functions and on the entropy as well as on the derivatives thereof. Thermodynamic equilibrium is determined by the variational equation

$$
\delta Q+\delta A=0
$$

which needs to be understood exactly in the old way; thereby also constraints can affect the thermal behavior of the medium, i. e. the function s.

However, the full significance of thermodynamics appears only, when one asserts to this ansatz the so-called first law [of thermodynamics], which states a generally valid connection of all effects including the temperature

82 H. v. Helmholtz, J. f. Math. 97 (1884), p. 111 = Wiss. Abhandl. III, p. 119 ff. Cf. IV 11, No. 23, Heun.

83 Cf. the applications of the very same method above in No. $7 e, p .654$ and No. $8 b, p .662$ as well as remark ${ }^{111}$ ). (Note of the translators: This corresponds to the footnote 84 in [42], i.e. «Cf. IV 12, P. Stäckel as well as for instance the presentation of the Jacobi-Hamilion theory in II A 5, No. 31, E. v. Weber. An extension to several independent variables is given by M. Born, Untersuch. über die Stabilität der elastischen Linie. Preisschrift, Göttingen 1906, Appendix.»)

84 See the articles V 3, No. 28ff. (Bryan) and IV 1, No. 48 (Voss).

85 For the foundations of common thermodynamics, which are just repeated in the following, cf. the article V 3 (Bryan). 
with a single function of the state variables of the kind as discussed in No. 7 for individual cases. ${ }^{86}$ Namely, if one considers in $\delta A$ only the internal effects within the medium, then $\delta Q+\delta A$ shall be up to the sign equal to the variation of a certain expression, the potential energy $\Phi$, depending for every medium in a characteristic way only on the respective deformation functions and the entropy. Concerning the form of $\Phi$, then the most simple case is, that $\Phi$ is a volume integral of a function $\varphi$ depending on $x, y, z$, the first derivatives thereof and $s^{87}$ :

$$
\delta Q+\delta A=-\delta \iiint_{\left(V_{0}\right)} \varphi\left(x, \ldots ; x_{a}, \ldots ; s\right) d a d b d c
$$

then especially for the temperature it follows

$$
\Theta=-\frac{1}{\varrho_{0}} \frac{\partial \varphi}{\partial s}
$$

which stands aside to the equations (4) of No. 7a [which] remain basically unchanged. From these equations again reciprocal relations of the kind

$$
-\varrho_{0} \frac{\partial \Theta}{\partial x_{a}}=\frac{\partial X_{a}}{\partial s}
$$

follow between a pair of thermal and elastic parameter - with a similar meaning, as discussed above for another case (No. 14, (4)).

To comment the impact of "entropy based" postulation of continuum thermo-mechanics presented in the previous paragraphs by Hellinger, it is enough to remark that in a great part of the subsequently cited literature the information about the discussed results seems to have been lost. This is probably another effect of the "erasure" operated by authoritativeness of the textbook by Truesdell and Noll [105]. We want to remark once more: if everybody (in a group) believes that a given textbook is a faithful picture of the state of the art, then what is not written there is simply "non-existing" in the dependent literature. Such literature uses the authoritative source as an "evangelium". 88

As an example of the effects of the erasure operated by Truesdell and Noll for what concerns variational principles, it is interesting to check what is written in the paper of Batra [10]. It has been published in 1989 in a prestigious journal, many years after the appearance of Hellinger's article. It has been carefully reviewed (received April the 24th and revised August the 29th of 1988). In this formula (1.1) the principle of virtual work (extended for thermoelastic bodies) is postulated for every part of a material body (called by Gurtin and Fried in [49] the non standard principle of virtual work). Remark that

i) Batra does not state that he invented such a version of the principle of virtual work

ii) Already in 1913, Hellinger (see [43]) has proven that it is equivalent to postulate the principle of virtual work for a whole body or for every part of a body.

iii) in Podio-Guidugli [90] it is stated that it is not easy to establish who formulated such nonstandard form of the principle of virtual work.

iv) Batra assumes as basic quantity the entropy, exactly as done in the just translated paragraphs by Hellinger.

Clearly, Batra (and all those involved in the reviewing process of his paper) considered definitively the textbook by Truesdell and Noll [105] as the ultimate source of all thermo-mechanical knowledge and he does not believe in the necessity to check other sources. Hellinger's article has been continuously available in every decent library in the United States and Europe and German is not a lost language. In fact:

86 For continuous media nominally P. Duhem has applied these fundamental approaches in various directions; one shall confer the summarizing presentation in his Traité d'Énergétique, T. II (Paris 1911), Chap. XIV.

87 For the special case of the pure theory of elasticity, this ansatz has been formulated originally by W. Thomson, Quart. Journ of Math. 1 (1857); cf. V 3, No. 21, Bryan.

88 We recall that there are many examples and a striking evidence about the widespread and uncritical acceptance of the authoritativeness of a source. When the source is reliable, the effects of its acceptance are very positive for the advancement of science. On the contrary, if the source is not reliable, then some parts of science are frozen or "rewound" back to a previous stage. An ancient example can be given by Fallopius who states in his Observationes Anatomicae «Herophili authoritas apud me circa res anatomicas est Evangelium» (see [113], p. xi) which can be translated as «The authority of Herophilus, for what concerns anatomy, is for me like the gospel». An alternative but similar statement of Fallopius, also cited on p. xi of [113], is «Quando Galenus refutat Herophilum, censeo ipsum refutare Evangelium medicum». The translation of this statement is given on p. 342 of [93] as «When Galen contradicts Herophilus, it is to me as if he contradicting medical gospel». 
i) formula (4) by Hellinger is exactly formula (1.4) before point (iii) in [10].

ii) formula (1.7) in [10] corresponds to the equation (4) of No. 7a (cf. [42]), which is simpler as it is totally Lagrangian and therefore not encumbered by Piola's transformations back and forth.

iii) formula (2) by Hellinger is equivalent to formula (3.6) in [10].

In conclusion, Hellinger is rediscovered and reformulated in the format established by Truesdell in the paper of Batra [10]. Of course the change of notation, the abundance of details and the paraded mathematical rigor and precision does not change the content of the presented subject: what is shown in [10] was exactly presented in Hellinger's article.

Often it is useful to introduce the temperature $\Theta$ as determining parameter instead of s; according to the form, this corresponds again with the canonical transformation applied in No. $7 e$ (p. 654): If one computes s as a function of $\Theta$ using (4) and if one determines therewith

$$
\psi=\varphi+\varrho_{0} \Theta s=\psi\left(x, \ldots ; x_{a}, \ldots ; \Theta\right),
$$

then one obtains instead of (3) for all arbitrary variations $\delta x, \delta y, \delta z$ and $\delta \Theta$ the identity:

$$
-\iiint_{(V)} s \delta \Theta \varrho d V+\delta A=-\delta \iiint_{\left(V_{0}\right)} \psi d a d b d c .
$$

One denotes $\psi$ the "thermodynamic potential for a given state of deformation"; if one considers at the same time also the transformation of No. $7 \boldsymbol{e}$, which substitutes the deformation quantities with the stress components, then one obtains the other types of thermodynamic potential in complete analogy to the common considerations of thermodynamics of systems with finitely many degrees of freedom. ${ }^{89}$

By specializing on the right hand side of (3) the appearance of the deformation quantities in a convenient way, one obtains the thermodynamic fundamentals for the before treated individual fields; Thereby the way how $s$ in $\varphi($ or $\Theta$ in $\psi)$ is related with the particular deformation quantities determines certainly the thermal effect of the individual kinds of deformation or the kind of deformation, which is caused by thermal effects. These relations have been studied in many ways for the theory of elasticity and hydrodynamics. ${ }^{90}$

Very similar considerations to those previously formulated in [10] can also be developed for instance for the paper of Podio-Guidugli [89].

Hellinger's presentation of the principle of virtual work for the case of thermoelasticity is rediscovered again nearly one century later. Remark that Hellinger attributes such a formulation to Duhem, Voigt and Hamel (see footnote 90). All considerations developed by Truesdellians about thermodynamic potentials and their relationships are easily described by Hellinger. See for instance the considerations leading to the unlabeled equation before formula $(3)^{\prime}$ in Hellinger. The canonical transformation described by Hellinger shows the easy equivalence between the results presented in [10] and those presented in [89]. The reader will remark that in the list of references of this last paper both Hellinger's article and [10] do not appear.

In (3) one has used for the potential energy $\Phi$ also other approaches studied in No. 7, where to the former formulas now the consideration of the dependence on $s$ is added anew. Besides the integrals of the kind No. 7, (7), which P. Duhem ${ }^{91}$ has used frequently for this kind of applications, let us highlight here the case, that $\Phi$ has as a summand a surface integral for instance over the interface between different media contained in $V$; accordingly, one needs to assume on this surface also a surface density of the entropy and consequently a surface integral as a contribution to the heat supply. This fundamental approaches represent the thermal effects of capillarity ${ }^{92}$.

In this last passages of No. 8, the senior author learnt that, without knowing it, in his first papers [29, 32-34] he was following a research flow started by Minkowski. Unfortunately, he cannot judge presently the actual originality of his own contribution, which should be assessed by carefully reading the papers cited by Hellinger in this context. However, it has to be remarked that the aforementioned papers were published in a rather selective journal and that they were followed

\footnotetext{
89 See V 3, No. 16 (Bryan).

90 Cf. e. g. Voigt, Kompendium I, p. 523 ff.; Voigt, Lehrbuch der Kristallphysik, Leipzig 1910, p. 276ff., p. 763ff.; Duhem, Traité d'énergétique II, Paris 1911, p. 115ff.; G. Hamel, Elementare Mechanik, Leipzig 1912, p. 571 ff.

91 See in particular Ann. Éc. Norm. (3) 10 (1893), p. $183 f f$. and 21 (1904), p. $99 f f$. and Traité, op. cit. ${ }^{86}$

92 Cf. V 9, No. 18 (Minkowski).
} 
by papers inspired on the same principles and methods (see e.g. $[6,48,61]$ ). Once more one has to remark that linguistic barriers and the authority of an incomplete textbook (again Truesdell and Noll [105]) did oblige subsequent researches to "rediscover" ideas and most likely results already available.

The further development of these thermodynamic approaches follows in the way that one let appear in the fundamental equation new physical parameters describing the constitution of the considered medium. It is enough to mention here as an example that by adding the electric field strength as in No. 14, (2) one is led to the occurrence of pyroelectricity in crystals, [i.e.] the interaction between heat, pressure and electric excitation. ${ }^{93}$

Finally, the thermochemical studies following J. W. Gibbs ${ }^{94}$ have to be mentioned which are based on the perception of various media occupying the same space simultaneously, whose state variables appear in $\varphi$ at once; here, certainly one has restricted oneself throughout to the case of finitely many degrees of freedom: one assumes the individual media (phases) to be homogeneous, such that their state is characterized by a series of variables not depending on the position anymore. ${ }^{95}$

\section{Annotated translation of No. 16 (pp. 685-694)}

16. Relations to the theory of relativity. At the end, the question shall be incorporated, which already has been touched repeatedly once in a while, how the various fundamental approaches of the mechanics of continua behave under the transformation of the used coordinate system; from here, also the relation to the foundations of the modern theory of relativity are established.

Our perception of the homogeneity and isotropy of the ordinary space demands at first that the laws of every physical process remain unchanged if one relates them to another orthogonal coordinate system and [if], at the same time, [one] subjects all quantities involved in the process to the corresponding transformation; it is said briefly, that the entire physics is invariant with respect to the group of all orthogonal coordinate transformations of the ordinary geometry, the so-called "basic group" or "Euclidean group". Herefrom it follows in particular, that the virtual work of all internal effects within a continuous system necessarily vanishes for a virtual displacement corresponding to an infinitesimal change of the coordinate system, or that the total potential of these effects remain unchanged for any such displacement of the continuum, i.e. [that the potential] is a euclidean potential in the sense of E. und F. Cosserat (cf. No. 7b, p. 650).

In the previous sentences Hellinger reformulates what Paul Germain [55] called the "axiom of internal forces" or "of internal effects" when considering the preceding French publication [54]. The reader can verify (see [26]) that already Gabrio Piola had formulated such an axiom in continuum mechanics. The critical analysis of such an axiom is the standard starting point of all modern presentation of relativistic theories. The fundamental contribution to the invariance requirements of the laws of physics due to the Cosserat brothers is also recognized by Hellinger here: the fundamental contributions to this subject by Emmy Noether are close to come, and the article by Hellinger shows how the mathematical and physical milieu in Germany was ready to her revolutionary contributions.

In a similar way, one can ask in kinetics if there are also transformations of the time variable $t$ or even simultaneous transformations of time and space variables which leave the physical laws unchanged. If one bases on the fundamental laws of kinetics in the original form (No. 5, (1), (5), (4), (6)), then it follows, that a displacement of the origin in the computation of time

$$
\bar{t}=t+\beta
$$

as well as a uniform motion of the orthogonal coordinate system parallel to itself

$$
\bar{x}=x+\alpha_{1} t, \quad \bar{y}=y+\alpha_{2} t, \quad \bar{z}=z+\alpha_{3} t
$$

do not change the kinetic terms essentially; only the time derivatives of 1. order, for instance the kinetic energy $T$, are modified at first by the substitution (2), but one sees easily that the additional terms vanish for the variation and thus the laws of motion remain unchanged. Hence, the theorems of the mechanics of continua are in a certain range invariant with respect to a ten-parameter group of linear transformations in space and time

93 See V 16, No. 11, F. Pockels.

94 Trans. Connect. Acad. III (1876-1878) = Scient. Papers I (1906), p. 55.

95 Cf. V 3, No. 26, (Bryan) and IV 11, No. 22-24 (K. Heun). 
coordinates $^{96}$, which are composed of an orthogonal coordinate transformation, a parallel displacement of the system of axes with constant velocity, as well as the changes of the origin in the computation of time; the general substitution of this so-called Galilean or Newtonian group formulates as:

$$
\begin{aligned}
& \bar{x}=\alpha_{11} x+\alpha_{12} y+\alpha_{13} z+\alpha_{1} t+\beta_{1} \\
& \bar{y}=\alpha_{21} x+\alpha_{22} y+\alpha_{23} z+\alpha_{2} t+\beta_{2} \\
& \bar{z}=\alpha_{31} x+\alpha_{32} y+\alpha_{33} z+\alpha_{3} t+\beta_{3} \\
& \bar{t}=t+\beta
\end{aligned}
$$

where the 9 quantities $\alpha_{i k}$ form an orthogonal system of coefficients. The substitutions (3) have the characteristic property to leave the differential $d t$ unchanged, but when $d t=0$, then also the square of the line element $d x^{2}+d y^{2}+d z^{2}$ [remains unchanged]. In mechanics also the extended twelve-parameter group [obtained by] adding all similarity transformations of the space on the one hand and of the time axis on the other hand is of particular importance; its application does not leave the physical quantities to be invariant, but relates their dimensions with respect to the unit length and time. ${ }^{97}$

In the previous paragraphs, Hellinger reports in a pedagogical way the fundamental invariance ideas on which variational postulations of mechanics are based. Correctly, he attributes to the Cosserat brothers the Lagrangian formulation of the invariance of the laws of physics under the transformations of coordinate systems. The heuristic role of variational methods, also in relativity, are widely recognized in the literature. We limit ourselves to cite here the interesting work [14] and the fundamental textbook [57], where the role of Levi-Civita (and of the mathematical tools which he, together with Ricci Curbastro, made available to mathematical physics) in the variational invention of relativity was highlighted.

Remark that the results presented by Hellinger are presented nearly verbatim for instance in [75] using a less sophisticated notation and approach. Indeed Møller imposes the invariance of the light speed for change of observer and from this he deduces the form of Lorentz transformations. He also postulates a form of balance of forces and then imposes its invariance under Lorentz transformation. It seems to us that obliterating the original discovering process in textbooks is not a really fruitful procedure. The reader will check easily also that in Noll [78] the contents of Hellinger's article are reformulated in a different notation.

In the modern development of optics and electrodynamics the fact that definitely not all laws of physics show this invariance with respect to the Galilean group has gained particular importance. This can be achieved on the one hand that according to the general foundations of No. $5 \mathrm{~d}$ and $7 f$ completely different kinetic terms as in classical mechanics determine the process, or on the other hand also thereby that for an unchanged ansatz a physical circumstance suggests for certain quantities a different interpretation and consequently a different treatment under transformations. Thus, for instance the fundamental equations of optics (3a) of No. 18, as they emerge exactly from the usual ansatz of d'Alembert's principle, must be invariant with respect to Galilean transformations, if one transforms only the $x-y$-z-space and [if one lets] unchanged $a, b, c$ as parameters characterizing every substantial particle. Contrary to this, optics gives rise to consider the light vector $u, v, w$ as a function of the point $a, b, c$ and to subject these variables $a, b, c$ accordingly to the Galilean transformation (2); thereupon after the transformation, the time derivative for constant $\bar{a}=a+\alpha_{1} t, \ldots$ has to be carried out, and in this sense the equations of optics are not anymore invariant.

If one determines however those linear transformations of the variables $a, b, c, t$ for which the fundamental laws of optics remain invariant in this sense ${ }^{98}$, then transformations emerge being denoted as Lorentztransformations according to H. Poincaré's ${ }^{99}$ suggestion, whose fundamental relevance for electrodynamics and physics has been approved by the studies of H. A. Lorentz ${ }^{100}$, A. Einstein ${ }^{101}$, H. Poincaré ${ }^{99}$, H. Minkowski ${ }^{102}$.

96 Cf. hereto the explanation in IV 1, No. 13-17, Voss.

97 Cf. about this IV 1, No. 10, Voss.

98 W. Voigt, Nachr. Ges. d. W. Göttingen 1887, p. 41.

99 H. Poincaré, Rendic. Circ. mat. Palermo 21 (1906), p. 129.

100 Versuch einer Theorie der elektrischen und optischen Erscheinungen in bewegten Körpern, § 89-92 (Leiden 1895). Amsterdam Acad. Sc. Proc. 6 (1904), p. 809. Published in Heft 2 of "Fortsch. d. math. Wissenschaften" (Leipzig 1913; hrsg. v. O. Blumenthal).

101 A. Einstein, Ann. d. Phys. (4) 17 (1905), p. 891. Published at the same place.

102 H. Minkowski, a) Die Grundgleichungen für die elektrodynamischen Vorgänge in bewegten Körpern, Nachr. Ges. d. W. Göttingen, math.-phys. Kl., 1908, p. 53 = Math. Ann. 68 (1910), p. 472; also published in Fortschr. d. math. Wiss. (Leipzig 1910), Heft 1. b) Raum und Zeit, Jahresber. d. D. M. V. 18 (1909), p. 75 = Phys. Z. 10 (1909), p. 104; also separately Leipzig 1909 and in the number referred to in 100). 
There are these "affinities" of the four-dimensional $x$-y-z-t-space, the Minkowskian "world":

$$
\begin{aligned}
& \bar{x}=\alpha_{11} x+\alpha_{12} y+\alpha_{13} z+\alpha_{14} t+\alpha_{15} \\
& \bar{y}=\alpha_{21} x+\alpha_{22} y+\alpha_{23} z+\alpha_{24} t+\alpha_{25} \\
& \bar{z}=\alpha_{31} x+\alpha_{32} y+\alpha_{33} z+\alpha_{34} t+\alpha_{35} \\
& \bar{t}=\alpha_{41} x+\alpha_{42} y+\alpha_{43} z+\alpha_{44} t+\alpha_{45},
\end{aligned}
$$

which transform the differential form $d x^{2}+d y^{2}+d z^{2}-c^{2} d t^{2}$ (in which c denotes the speed of light) into itself:

$$
d \bar{x}^{2}+d \bar{y}^{2}+d \bar{z}^{2}-c^{2} d \bar{t}^{2}=d x^{2}+d y^{2}+d z^{2}-c^{2} d t^{2}
$$

and for which moreover it holds:

$$
\frac{d \bar{t}}{d t}=\alpha_{44}>0
$$

they form again a ten-parameter group, the Lorentz group. If one notes, that due to (5) the transformation (4) represents an orthogonal substitution in the space of coordinates $x, y, z, c t \sqrt{-1}$, then one can easily give the relation for the coefficients of (4) and the invariants of this group. ${ }^{103}$ After all one can characterized a somehow more encompassing "extended Lorentz group" geometrically thereby, that it transforms into itself the quadratic area $x^{2}+y^{2}+z^{2}-c^{2} t^{2}=0$ of the indefinitely far-away three-dimensional shape of the four-dimensional $x$ - $y$ $z$-t-space, and therefore one can take the theory thereof from known studies of projective or affine geometry. ${ }^{104}$ This extended group contains eleven instead of ten parameters, and their transformations fulfill the identity (5) only up to a constant factor; If one determines this [factor] for instance by the determinant condition

$$
\left|\alpha_{i k}\right|=1 \quad(i, k=1,2,3,4)
$$

then [the group] decomposes into two separate continua [i.e. connected components], from which one is identical with the Lorentz group characterized by (6). The eleventh parameter of the extended group corresponds to a change of the measuring unit in the $x-y$-z-t-space; in [this space] there is only one measuring unit available, since space and time coordinates are related by the requirement of the invariance of the form (5), i. e. by fixing the speed of light, while for an extension of the Galilean group by two parameters, we could separately decide about time and spatial unit. ${ }^{105}$

If one lets c converge towards $\infty$, then the extended Lorentz group changes into the totality of linear transformations, which transform into itself the quadratic curve in the infinity of the $x-y$-z-t-space determined by the two equations $x^{2}+y^{2}+z^{2}=0, t=0$ (this is the imaginary spherical circle of the spaces $t=$ const.); this is just the extended Galilean group ${ }^{106}$, and consequently the Galilean group is the limit case of the Lorentz group for indefinitely growing constant c. This has motivated Minkowski to give to the so-called relativity principle, which has been stated at first as a requirement of the invariance with respect to the Lorentz group for the laws of electrodynamics, a further range of validity as "postulate of the absolute world"107: What seems at first as invariance with respect to the Galilean group, is in fact only an empirical approximation of the exact invariance with respect to the Lorentz group with a very large c compared to the usually appearing velocities.

The foundations of the dynamics of a continuum which respects this relativity postulate are included in the previously formulated general forms; we only have to choose all relevant state functions as invariants or covariants of the Lorentz group. If the motion of the continuum is given again as in No. 2, (5), then the formulas arrange more homogeneously when one introduces for every particle $a, b, c$ a "local time"

$$
\tau=\tau(a, b, c, t)
$$

as function of time $t$; by setting for the sake of symmetry

$$
\begin{aligned}
& x=x_{1}, \quad y=x_{2}, \quad z=x_{3}, \quad t=x_{4}, \\
& a=\xi_{1}, \quad b=\xi_{2}, \quad c=\xi_{3}, \quad \tau=\xi_{4},
\end{aligned}
$$

\footnotetext{
103 H. Minkowski, ${ }^{102}$ a) § 5; cf. also A. Sommerfeld, Ann. d. Phys. (4) 32 (1910), p. 749; 33 (1910), p. 649

104 F. Klein, Die geometr. Grundlagen der Lorentzgruppe, Jahresber. d. D.M.V. 19 (1910), p. 281.

105 Cf. F. Klein, op. cit., p. $295 f$.

106 F. Klein, op. cit., p. $291 f$.

107 H. Minkowski, ${ }^{102}$ a) Anhang; b) Cap. I, II.
} 
then the equations of motion write as

$$
x_{i}=x_{i}\left(\xi_{1}, \xi_{2}, \xi_{3}, \xi_{4}\right) \quad(i=1,2,3,4)
$$

they represent for varying $\xi_{4}$ a system of curves (world lines) simply covering the four-dimensional space [. Curves] whose whole courses give a complete picture of the motion. ${ }^{108}$

An essential supplement of the relativity postulate forms the requirement, that all generally possible velocities are below the speed of light, i. e. when we generally set

$$
\frac{\partial x_{i}}{\partial \xi_{k}}=x_{i k} \quad(i, k=1,2,3,4),
$$

that

$$
x_{14}^{2}+x_{24}^{2}+x_{34}^{2}<c^{2} x_{44}^{2}
$$

or - geometrically spoken - that every tangent to a world line lies within the cone of the directions $d x^{2}+d y^{2}+$ $d z^{2}=c^{2} d t^{2}$. Equivalently to this is the fact that one can "transform to rest" every particle for every time by a suitable Lorentz transformation (4), i. e. that one can change over to a new coordinate system $\bar{x}_{i}$, in which for similar labeling as in (7a) for the considered tuple $\xi_{1}, \ldots \xi_{4}$

$$
\bar{x}_{14}=\bar{x}_{24}=\bar{x}_{34}=0 .
$$

All possible "transformations of rest" differ, apart from the arbitrarily remaining quantities $\alpha_{15}, \ldots \alpha_{45}$, only by an ordinary orthogonal transformation of the three coordinates $\bar{x}_{1}, \bar{x}_{2}, \bar{x}_{3}$; in the different "coordinate systems of rest" consequently all deformation quantities of first order $\bar{x}_{i k}$ possibly will be different from each other, whereas the effective "shape change components" (No. 9, (1))

$$
\begin{aligned}
e_{i} & =\frac{1}{2}\left(\bar{x}_{1 i}^{2}+\bar{x}_{2 i}^{2}+\bar{x}_{3 i}^{2}-1\right), \\
g_{i k} & =\bar{x}_{1 i} \bar{x}_{1 k}+\bar{x}_{2 i} \bar{x}_{2 k}+\bar{x}_{3 i} \bar{x}_{3 k}
\end{aligned} \quad(i, k=1,2,3)
$$

being invariant with respect to the orthogonal coordinate transformations will be independent of the specially chosen transformations of rest. If one thinks of $\bar{x}_{i k}$ being substituted by their expressions of the original motion, then these deformations of rest are the only invariants of first order, which the system of world lines (7) shows with respect to the Lorentz group; they are likewise also independent of the arbitrary choice of the parameter $\xi_{4}=\tau .{ }^{109}$

The patient reader will find a treatment very similar to the one presented in the previous paragraphs in [96]. Patience will be needed in order to adapt to the change of notation, which, once more, is not changing the substance of presented ideas. Remark that Hellinger attributes to Minkowski all the presented results, which are presented in English many decades after in a slightly different form (see e.g. Noll [78]).

A virtual variation of the motion of the continuum is represented now by the four functions $\delta x_{i}(i=1, \ldots 4)$; since the variable $\xi_{4}=\tau$ is arbitrary, this implies that the motion of the continuum itself, i. e. the system of world lines, [is described] only [by] three arbitrary functions. The virtual work of any volume forces applied in the interval $\tau_{1} \leqq \tau \leqq \tau_{2}$ is then assumed to be given by the expression

$$
\delta A=\int_{\tau_{1}}^{\tau_{2}} d \tau \iiint_{\left(V_{0}\right)} \sum_{i=1}^{4} X_{i} \delta x_{i} \varrho_{0} d \xi_{1} d \xi_{2} d \xi_{3} .
$$

Relativistic continuum mechanics is formulated in 1913 by Hellinger as a well-established theory. He bases his presentation on the postulation of the form of virtual work (remark that he uses a "local time" for every particle, i. e. the time used by an observer for which the particle is "at rest", see e.g. [75]) and on the specification of the set of admissible motions and admissible variations of motions. This point of view is not often accepted in the subsequent relevant literature. We list here some comments concerning some randomly chosen subsequent papers or textbooks, which we believe are representative of the whole body of publications posterior to Hellinger's in relativistic continuum mechanics:

\footnotetext{
108 H. Minkowski, ${ }^{102}$ b).

109 M. Born, Ann. d. Phys. (4) 30 (1909), p. 1; especially § 2. - G. Herglotz, Ann. d. Phys. (4) 36 (1911), p. 493; especially § 1, 2.
} 
i) in Møller [75], the theory of relativistic deformable bodies is based on a reformulation of the Cauchy tetrahedron argument and on an "ad hoc" postulation of balance equations - this time, energy balance is not a trivial consequence of the balance of forces as in classical mechanics: mass can evolve!

ii) In [41], the approach presented in Møller is enlarged to incorporate coupled phenomena of mechanics and electrodynamics. The scheme ("corso" and "ricorso") which can be found in the publication of [106] and the paper [100] is observed (although in slightly different form) in the passage from the previous textbook [41] to the fundamental review paper [72], where Maugin is the only author. In this last review the presented subject is extremely lucid, elegant and concise: Maugin refrains from including relativistic invariance to work functionals, however, the whole mathematical structure needed is carefully introduced and described. Maugin can freely base his postulation on the principle of virtual work in the paper which he is authoring alone, while Toupin must regard the principle of virtual work as a secondary concept: indeed he is publishing in a journal controlled by Truesdell. Moreover, Toupin must limit himself to consider the only case of second gradient continua in which balance of force and balance of couples can produce the correct evolution equations.

iii) Most likely due to the influence of Sedov (cf. [27]) in the Soviet science, the paper [60] presents a clear and elegant formulation based on variational principles.

iv) In Landau and Lifshitz [70], when dealing with relativistic fluid dynamics the variational approach is assumed to be granted (as it is discussed in the other volumes, addressing the more general conceptual issues), while in [98] the variational point of view is simply ignored and the presentation is based on the standard list of balance equations, suitably adapted.

v) In [7] the fundamental differential equations are postulated without any comment and they are studied from a mathematical point of view.

vi) In [59] the whole set of Euler-Lagrange equations (deduced by means of variational principles as in Hellinger) is postulated "a posteriori" to adhere to the Cauchy-Truesdellian logical paradigm.

vii) In [96] one finds an exercise in the style of Noll: what is presented therein is the reformulation of perfectly known matter simply making the notation more complex and painfully showing obvious statements in a slightly more general form than usual.

viii) A very interesting gallery of mathematical and physical concepts is presented in [58], clearly inspired by the flow of ideas presented by Hellinger. Although the mathematics is really rigorous and modern and the geometrical frame sound, it is astonishing to see how the clear variational frame in which these ideas naturally arise could be removed. While in a variational context the concept of hyperstress arises naturally (see the whole article by Hellinger for being persuaded of this), this concept can be understood only "a posteriori" within the framework of balance of forces and energy. Once more one can conclude that a wrong postulation choice can dramatically slow down the advancement of knowledge.

ix) In [46] a compromise is attempted. Indeed, not to openly challenge the dominant (in Italian mechanics milieu of the time) Truesdellian paradigm, after the description of the kinematical basics, the authors postulate balance equations, as usual getting them out of the blue. However few pages later they present the corresponding variational principles and quietly show to belong to the strong traditional Italian school, founded by Lagrange and continued by Piola. The reader is referred here to the discussion in [43] about the pseudo-Aristotelian Mechanical Problems.

Thereby by introducing the factor $\frac{d \tau}{d t}=\frac{1}{x_{44}}$ to $X_{1}, X_{2}, X_{3}$, they denote similarly to No. 3a, $p$. 613, the force components computed with respect to unit mass of the undeformed medium in the $\xi_{1}-\xi_{2}-\xi_{3}$-space; if one notices furthermore, that a variation for which at every point

$$
\delta x_{1}: \delta x_{2}: \delta x_{3}: \delta x_{4}=x_{14}: x_{24}: x_{34}: x_{44}
$$

implies only a displacement of the world line in itself, thus [implies] a change of the parameter $\tau$, and that for [this variation] $\delta A$ consequently has to vanish identically, then it follows that

$$
-X_{4}=\frac{1}{x_{44}} \sum_{i=1}^{3} x_{i 4} X_{i}=X_{1} \frac{d x_{1}}{d t}+X_{2} \frac{d x_{2}}{d t}+X_{3} \frac{d x_{3}}{d t}
$$


- again up to the factor $\frac{1}{x_{44}}$ - which denotes the work done at the mass unit of the undeformed medium in the unit of time. ${ }^{110}$

Analogously (cf. No. 5, (10)), the integral

$$
\delta A_{1}=-\int_{\tau_{1}}^{\tau_{2}} d \tau \iint_{\left(V_{0}\right)} \sum_{i, k=1}^{4} X_{i k} \frac{\partial \delta x_{i}}{\delta \xi_{k}} d \xi_{1} d \xi_{2} d \xi_{3}
$$

is assumed as work [expression] for any stresses applied at the continuum for a virtual displacement [i.e.] the "stress action" of H. Minkowski ${ }^{111}$; since for the virtual displacement (10) also $\delta A_{1}$ has to vanish identically, the [following] identities are obtained

$$
\sum_{i=1}^{4} x_{i 4} \sum_{k=1}^{4} \frac{\partial X_{i k}}{\partial \xi_{k}}=0
$$

in the interior of $V_{0}$ and

$$
\sum_{i=1}^{4} x_{i 4} \sum_{k=1}^{3} \frac{\partial \omega}{\partial \xi_{k}} X_{i k}=0
$$

at the boundary, provided that the equation thereof is ${ }^{112}$

$$
\omega\left(\xi_{1}, \xi_{2}, \xi_{3}\right)=0
$$

The transformations applied already several times allow to extract the equations of motion from the generalized Hamilton's principle, which demands the vanishing of

$$
\delta A+\delta A_{1}=0
$$

for all arbitrary virtual displacements [which] vanish identically only for $\tau=\tau_{1}$ and $\tau=\tau_{2}$ :

$$
\varrho_{0} X_{i}+\sum_{k=1}^{4} \frac{\partial X_{i k}}{\partial \xi_{k}}=0 \quad \text { in } V_{0},
$$

$$
(i, 1, \ldots 4)
$$

$$
\sum_{k=1}^{3} \frac{\partial \omega}{\partial \xi_{k}} X_{i k}=0 \quad \text { on the boundary of } V_{0} .
$$

Due to the identities (11a), (11b) each one of these four equations is dependent on the other three. Analogously to before (No. $3 c, p .617 f$.) in (11) one can introduce instead of the $\xi_{i}$ the $x_{i}$ as independent variables, and one obtains then a form of the equations of motion corresponding to the equations (5) of No. 3, as they have been given by Minkowski. ${ }^{113}$

At first, the class with dependence of the stress components on the motion (7) can be chosen completely freely, as long as the relations (11a), (11b) are fulfilled; here only the potential-based approach No. 7, (26) is considered, which leads to the transmission of the formulas from the ordinary theory of elasticity (No. 9, (2), (3)) to the theory of relativity of the Lorentz group given by $G$. Herglotz ${ }^{114}$. Let therefore

$$
-\delta A_{1}=+\delta \Phi=\delta \int_{\tau_{1}}^{\tau_{2}} d t \iiint_{\left(V_{0}\right)} \varphi\left(x_{i k}\right) d \xi_{1} d \xi_{2} d \xi_{3},
$$

110 H. Minkowski, ${ }^{102}$ a) Anhang; G. Herglotz, op. cit., p. 506.

111 H. Minkowski, ${ }^{102}$ a) Anhang, formula (17)

112 G. Herglotz, op. cit., p. $506 f$.

113 H. Minkowski, 102 a) appendix, formula (20). The equations appear in slightly modified form, since for him the parameter $\tau$ is always the "proper time" defined by (15) and thus for a variation it does appear always a constraint and a Lagrange multiplier.

114 G. Herglotz, op. cit. p. $503 \mathrm{ff}$. - Cf. also for the special case of hydrodynamics - analogous to No. 10 - the foundations of E. Lamla Ann. d. Phys. (4) 37 (1912), p. 772. 
where $\varphi$ may depend only on the first derivatives of the motion; then $\Phi$ remains unchanged for all Lorentz transformations only if $\varphi$, up to the factor $x_{44}$, depends merely on the six rest deformations (8):

$$
\varphi=\varphi\left(e_{1}, e_{2}, e_{3}, g_{12}, g_{23}, g_{31}\right) \cdot x_{44} .
$$

Comparing (13) and (11) it follows now that

$$
X_{i k}=\frac{\partial \varphi}{\partial x_{i k}},
$$

and the substitution of these values into (12a), (12b) leads to the fundamental equations given by Herglotz. ${ }^{115}$

Minkowski pushed the analogy with the classical Hamilton's principle further by separating from the work expressions, in which here from the beginning the kinetic terms are contained, generally a purely kinetic part. ${ }^{116}$ If one considers in the neighborhood of a certain point $x_{i}$ in a corresponding rest coordinate system $\bar{x}_{i}$ and [if one] measures volume and mass of the medium in this $\bar{x}_{1}-\bar{x}_{2}-\bar{x}_{3}-$ space, then the mass density $\bar{\varrho}$ which is given for the point $\bar{x}_{i}$ is called rest density at this point. Minkowski lets then add the variation of the so-called mass action, [i.e.] the integral extended over the considered four-dimensional space

$$
P=\iiint \int \bar{\varrho} d x d y d z d t
$$

to (12), whereas during the variation the mass has to be kept constant. If one uses now a special proper time $\tau$, namely the one, which satisfies the relation

$$
c^{2}\left(\frac{\partial x_{4}}{\partial \tau}\right)^{2}-\left(\frac{\partial x_{1}}{\partial \tau}\right)^{2}-\left(\frac{\partial x_{2}}{\partial \tau}\right)^{2}-\left(\frac{\partial x_{3}}{\partial \tau}\right)^{2}=c^{2},
$$

then $\delta P$ becomes up to boundary terms equal to

$$
\iiint \int \bar{\varrho}\left(\frac{\partial^{2} x_{1}}{\partial \tau^{2}} \delta x_{1}+\frac{\partial^{2} x_{2}}{\partial \tau^{2}} \delta x_{2}+\frac{\partial^{2} x_{3}}{\partial \tau^{2}} \delta x_{3}-\frac{\partial^{2} x_{4}}{\partial \tau^{2}} \delta x_{4}\right) d x d y d z d t,
$$

and the factors of $\delta x_{i}$ are added - in complete analogy to the fundamental equations of the Newtonian Mechanics - to the equations (12). M. Born ${ }^{117}$ has shown, how one can introduce the mass factor also as Lagrange multiplier of the constraint (15).

(Completed in August 1913.)

\section{Conclusions: Historia magistra vitae}

Historia magistra vitae ${ }^{118}$, or what we learn by reading Hellinger. In the perspective of advancement of science and progress of mankind, the life's problems and crises which had to be solved by Nicolò Tartaglia or Giambattista Vico are not relevant. However, these problems played a crucial role in choosing their way to contribute to the aforementioned progress. Tartaglia never managed to get a university chair and Vico did not manage to be promoted to the better paid chair of civil law. ${ }^{119}$ It has been proven that Tartaglia attributed to himself the translation by Luca Gaurico and William De Moerbeke and tried to imply that he could improve Archimedes himself. Vico most likely knew about Polybius and rephrased his historical theory in Italian most likely with some original contributions even if these contributions cannot be fairly evaluated as the

115 op. cit., p. $505 f$.

116 H. Minkowski, ${ }^{102}$ a) appendix, formula (7) to (14).

117 Ann. d. Phys. (4) 28 (1909), p. 571.

118 The complete dictum by Cicero can be found in paragraph 36 of the second book of De Oratore, [97] which is «Historia vero testis temporum, lux veritatis, vita memoriae, magistra vitae, nuntia vetustatis, qua voce alia nisi oratoris immortalitati commendatur?» which reads in English as: «And as History, which bears witness to the passing of the ages, sheds light upon reality, gives life to recollection and guidance to human existence, and brings tidings of ancient days, whose voice, but the orator's, can entrust her to immortality?» We simply suggest to read at the place of word "Orator" the word "Scholar". The source of Cicero is surely Polybius, The Histories, [79] cf. Book I, paragraph 35 «In these events there will be found by one who notes them aright much to contribute to the better conduct of human life».

119 However Vico's fame, subsequent to the publication of his works, allowed to his preferred last son to become his successor in the Chair of Rhetorics. 
greatest part of the Polybius' works are lost. These vicissitudes motivate the more or less deliberate blurring in referring about their primary sources. To the reader is left the imagination exercise needed to find similar motivations in the choices by Truesdell, Toupin and Noll. Actually, Hellinger's text is not lost and it is written in an easily understandable German. It proves that:

i) the main original contributions to mechanics were obtained starting from a variational postulation based on the principle of virtual work, or alternatively on the least action principle;

ii) a direct, although sometimes hidden, relation can be tracked from the works by D'Alembert, Euler and Lagrange up to Mindlin, Toupin and Sedov (see [27]) via Piola, Cosserat, Kirchhoff and Hellinger;

iii) modern computations and predictions based on numerical methods exploited greatly the mathematical tradition which is at the base of mechanics so that we can state that variational principles are the evergreen conceptual tools for engineering sciences. Some recent specific examples of this statement can be given by the results starting from the ideas by Sedov, Toupin and Germain presented in [1, 28, 40, 56, 84-88, 107-111]. In all these papers the power of variational principles allowed for the formulation of effective numerical methods capable to formulate careful predictions of strongly non-linear phenomena.

iv) the capacity of popularizing knowledge is important also in hard science and it is important that scientists may be capable to write their work at the same time in an understandable and precise way.

We greatly enjoyed to hear the calm but strong voice of the unfortunate Hellinger, a great mathematician who deserves to be regarded as a first class successor of Archimedes. Our understanding of continuum mechanics increased because of our translation and commentary work. Our hope is that our persuasion will be shared by the readers.

\section{References}

[1] J. J. Alibert and A. Della Corte, Second-gradient continua as homogenized limit of pantographic microstructured plates: a rigorous proof, Z. Angew. Math. Phys. 66(5), 2855-2870 (2015).

[2] T. Alts and K. Hutter, Continuum description of the dynamics and thermodynamics of phase boundaries between ice and water, part I. Surface balance laws and their interpretation in terms of three-dimensional balance laws averaged over the phase change boundary layer, J. Non-Equilib. Thermodyn. 13(3), 221-258 (1988).

[3] T. Alts and K. Hutter, Continuum description of the dynamics and thermodynamics of phase boundaries between ice and water, part II. Thermodynamics, J. Non-Equilib. Thermodyn. 13(3), 259-280 (1988).

[4] T. Alts and K. Hutter, Continuum description of the dynamics and thermodynamics of phase boundaries between ice and water, part III. Thermostatics and its consequences, J. Non-Equilib. Thermodyn. 13(4), 301-330 (1988).

[5] T. Alts and K. Hutter, Continuum description of the dynamics and thermodynamics of phase boundaries between ice and water, part IV. On thermostatic stability and well-posedness, J. Non-Equilib. Thermodyn. 14(1), 1-22 (1989).

[6] D. M. Anderson, P. Cermelli, E. Fried, M.E. Gurtin, and G. B. McFadden, General dynamical sharp-interface conditions for phase transformations in viscous heat-conducting fluids, J. Fluid Mech. 581, 323-370 (2007).

[7] A. M. Anile, Relativistic fluids and magneto-fluids: With Applications in Astrophysics and Plasma Physics, Cambridge Monographs on Mathematical Physics (Cambridge University Press, 1982).

[8] S. S. Antman, Nonlinear Problems of Elasticity, 2nd edition, Applied Mathematical Sciences, Vol. 107 (Springer, 2005).

[9] N. Auffray, F. dell'Isola, V. A. Eremeyev, and G. Rosi, Analytical continuum mechanics à la Hamilton-Piola least action principle for second gradient continua and capillary fluids, Math. Mech. Solid. 20(4), 375-417 (2013).

[10] G. Batra, On a principle of virtual work for thermo-elastic bodies, J. Elasticity 21(2), 131-146 (1989).

[11] E. Benvenuto, La scienza delle costruzioni e il suo sviluppo storico, La scienza delle costruzioni e il suo sviluppo storico (G. C. Sansoni, 1981).

[12] P. Casal and H. Gouin, Connection between the energy equation and the motion equation in Korteweg theory of capillarity, CR. Acad. Sci. II 300(7), 231-234 (1985).

[13] P. Casal and H. Gouin, A representation of liquid vapor interfaces by using fluids of 2nd grade, in: Annales de Physique, (1988), pp. 3-12.

[14] C. Cattani and M. Demaria, The 1915 epistolary controversy between A. Einstein and T. Levi-Civita, in: Presented at 4th Marcell Grossmann Meeting on the Recent Developments of General Relativity, Rome, Italy, 17-21 Jun. 1985, (1985).

[15] P. G. Ciarlet, A justification of the von Kármán equations, Arch. Ration. Mech. Anal. 73(4), 349-389 (1980).

[16] M. Clagett, Archimedes in the Middle Ages, Vol. 1.: The Arabo-Latin Tradition (The University of Wisconsin Press, Madison, 1964).

[17] M. Clagett, Archimedes in the Middle Ages, Vol. 2: The Translation from the Greek by William of Moerbeke (American Philosophical Society, Philadelphia, 1967).

[18] M. Clagett, Archimedes in the Middle Ages, Vol. 3: The fate of the medieval Archimedes 1300-1565 (American Philosophical Society, Philadelphia, 1978). 
[19] M. Clagett, Archimedes in the Middle Ages, Vol. 4: A Supplement on the Medieval Latin Tradition of Conic Sections (American Philosophical Society, Philadelphia, 1980).

[20] M. Clagett, William of Moerbeke: Translator of Archimedes, Proceedings of the American Philosophical Society 126(5), 356366 (1982)

[21] M. Clagett, Archimedes in the Middle Ages, Vol. 5: Quasi-Archimdean Geometry in the Thirteen Century (American Philosophical Society, Philadelphia, 1984).

[22] T. Davidson, Aristotle and Ancient Educational Ideals, Great educators, ed. by N. M. Butler (Scribner, 1892).

[23] F. dell'Isola, U. Andreaus, and L. Placidi, At the origins and in the vanguard of peridynamics, non-local and higher-gradient continuum mechanics: An underestimated and still topical contribution of Gabrio Piola, Math. Mech. Solid. 20(8) (2015).

[24] F. dell'Isola, H. Gouin, and G. Rotoli, Nucleation of spherical shell-like interfaces by second gradient theory: numerical simulations, Eur. J. Mech. B-Fluid 15(4), 545-568 (1996).

[25] F. dell'Isola, H. Gouin, and P. Seppecher, Radius and surface tension of microscopic bubbles by second gradient theory, CR. Acad. Sci. II 320(5), 211-216 (1995).

[26] F. dell'Isola, G. Maier, U. Perego, U. Andreaus, R. Esposito, and S. Forest, The complete works of Gabrio Piola: Volume I: Commented English Translation, No. 38 in Advanced Structured Materials (Springer, 2014).

[27] F. dell'Isola, A. Della Corte, and I. Giorgio, Higher-gradient continua: The legacy of Piola, Mindlin, Sedov and Toupin and some future research perspectives, Math. Mech. Solid. (2016).

[28] F. dell'Isola, A. Della Corte, L. Greco, and A. Luongo, Plane bias extension test for a continuum with two inextensible families of fibers: A variational treatment with lagrange multipliers and a perturbation solution, International Journal of Solids and Structures 81(3), 1-12 (2016).

[29] F. dell'Isola and D. Iannece, On phase transition in classical fluid mixtures with surface adsorption, Int. J. Eng. Sci. 27(9), 1069-1078 (1989).

[30] F. dell'Isola and W. Kosinski, Deduction of thermodynamic balance laws for bidimensional nonmaterial directed continua modelling interphase layers, Arch. Mech. pp.333-359 (1993).

[31] F. dell'Isola and W. Kosinski, The interface between phases as a layer, part II. A H-order model for two dimensional nonmaterial continua (2010).

[32] F. dell'Isola and A. Romano, On a general balance law for continua with an interface, Ric. Mat. pp. 325-337 (1986).

[33] F. dell'Isola and A. Romano, On the derivation of thermomechanical balance equations for continuous systems with a nonmaterial interface, Int. J. Eng. Sci. 25(11-12), 1459-1468 (1987).

[34] F. dell'Isola and A. Romano, A phenomenological approach to phase transition in classical field theory, Int. J. Eng. Sci. 25(1112), 1469-1475 (1987).

[35] E. J. Dijksterhuis, The Principal Works of Simon Stevin (Swets \& Zeitlinger, Amsterdam, 1955).

[36] E. J. Dijksterhuis, Simon Stevin: Science in the Netherlands around 1600 (Nijhoff, The Hague, 1970).

[37] E. J. Dijksterhuis, Archimedes, Princeton Legacy Library (Princeton University Press, Princeton, 1987).

[38] S. Drake, Cause, Experiment, and Science: A Galilean Dialogue incorporating a New English Translation of Galileo's "Bodies that Stay atop Water, or Move in it" (University of Chicago Press, Chicago, 1981).

[39] S. Drake and I. E. Drabkin, Mechanics in sixteenth-century Italy (University of Wisconsin Press, Madison, 1969).

[40] K. Enakoutsa, A. Della Corte, and I. Giorgio, A model for elastic flexoelectric materials including strain gradient effects, Mathematics and Mechanics of Solids 21(2), 242-254 (2016).

[41] A. C. Eringen and G. A. Maugin, Electrodynamics of Continua I: Foundations and Solid Media (Springer Science \& Business Media, 2012).

[42] S. R. Eugster and F. dell'Isola, Exegesis of sect. II and III.A from "Fundamentals of the mechanics of continua" by E. Hellinger, Z. Angew. Math. Mech. pp. 1-39, accepted 2017.

[43] S. R. Eugster and F. dell'Isola, Exegesis of the introduction and sect. I from "Fundamentals of the mechanics of continua" by E. Hellinger, Z. Angew. Math. Mech. 97(4), 477-506 (2017).

[44] L. Euler, Methodus inveniendi lineas curvas maximi minimive proprietate gaudentes sive solutio problematis isoperimetrici (Marcus-Michael Bousquet, 1744).

[45] E. Fermi, Thermodynamics, Dover Books on Physics (Dover Publications, 2012).

[46] G. Ferrarese and D. Bini, Introduction to relativistic continuum mechanics (Springer, 2008).

[47] G. A. Francfort and J. J. Marigo, Revisiting brittle fracture as an energy minimization problem, J. Mech. Phys. Solids 46(8), $1319-1342(1998)$

[48] E. Fried and M.E. Gurtin, A phase-field theory for solidification based on a general anisotropic sharp-interface theory with interfacial energy and entropy, Physica D 91(1-2), 143-181 (1996).

[49] E. Fried and M. E. Gurtin, Tractions, balances, and boundary conditions for nonsimple materials with application to liquid flow at small-length scales, Arch. Ration. Mech. An. 182(3), 513-554 (2006).

[50] G. Friesecke, R. D. James, and S. Müller, A hierarchy of plate models derived from nonlinear elasticity by gamma-convergence, Archive for Rational Mechanics and Analysis 180(2), 183-236 (2006).

[51] R. Gatignol and R. Prud'homme, Mechanical and thermodynamical modeling of fluid interfaces (World Scientific, Singapore, 2001).

[52] R. Gatignol and P. Seppecher, Modélisation of fluid-fluid interfaces with material properties, J. Mec. Theor. Appl. pp. 225-247 (1986).

[53] R. Gatignol, Asymptotic modelling of fluid-fluid interfaces, in: Dynamics of Multiphase Flows Across Interfaces, (Springer, 1996), pp. 14-27. 
[54] P. Germain, Sur l'application de la méthode des puissances virtuelles en mécanique des milieux, Cr. Acad. Sci. A, 1051-1055 (1972).

[55] P. Germain, The method of virtual power in continuum mechanics. part 2: Microstructure, SIAM J. Appl. Math. 25, 556-575 (1973).

[56] I. Giorgio, Numerical identification procedure between a micro-Cauchy model and a macro-second gradient model for planar pantographic structures, Z. angew. Math. Mech. 67(4), 95 (2016).

[57] H. Goldstein, C. Poole, and J. Safko, Classical Mechanics (Addison Wesley, 2002).

[58] F. Gronwald and F. W. Hehl, Stress and hyperstress as fundamental concepts in continuum mechanics and in relativistic field theory, arXiv preprint gr-qc/9701054 (1997).

[59] R. A. Grot and A. C. Eringen, Relativistic continuum mechanics part I - mechanics and thermodynamics, Int. J. Eng. Sci. 4(6), $611-638(1966)$.

[60] V.T. Gurovich and K. P. Staniukovich, On the application of general variational principles in the relativistic mechanics of an ideal fluid, J. Appl. Math. Mech. 29(1), 18 - 25 (1965).

[61] M. Gurtin, J. Weissmüller, and F. Larche, A general theory of curved deformable interfaces in solids at equilibrium, Philos. Mag. A 78(5), 1093-1109 (1998).

[62] T.L. Heath, The Method of Archimedes, Recently Discovered by Heiberg: A Supplement to the Works of Archimedes (Cambridge: at the Universtiy Press, London, 1912).

[63] J. L. Heiberg, Archimedis Opera Omnia: Cum Commentariis Eutocii, Vol. I-III (B. G. Teubneri, Leipzig, 1880-1881).

[64] J. L. Heiberg, Archimedis Opera Omnia: Cum Commentariis Eutocii, Bibliotheca Scriptorum Graecorum et Romanorum Teubneriana, Vol. I (B.G. Teubneri, Stuttgart, 1910).

[65] J. L. Heiberg, Archimedis Opera Omnia: Cum Commentariis Eutocii, Bibliotheca Scriptorum Graecorum et Romanorum Teubneriana, Vol. II (B.G. Teubneri, Stuttgart, 1913).

[66] Y. Ignatieff, The Mathematical World of Walter Noll: A Scientific Biography (Springer, Berlin, 2013).

[67] G. Kirchhoff, Über das Gleichgewicht und die Bewegung einer elastischen Scheibe, J. reine angew. Math. 40, 51-88 (1850).

[68] W. Kosiński, Field Singularities and Wave Analysis in Continuum Mechanics, Ellis Horwood series in mathematics and its applications (Ellis Horwood, 1986).

[69] L. D. Landau and E. M. Lifshitz, Continuum Electrodynamics, Course of Theoretical Physics, Vol. 8 (Butterworth-Heinemann, 1982).

[70] L. D. Landau and E. M. Lifshitz, Fluid mechanics (Butterworth-Heinemann, 1987).

[71] J. T. Lloyd, Background to the joule-mayer controversy, Notes and Records of the Royal Society 25(2), 211-225 (1970).

[72] G. A. Maugin, The method of virtual power in continuum mechanics: Application to coupled fields, Acta Mechanica 35(1), 1-70 (1980).

[73] G. A. Maugin, Continuum Mechanics of Electromagnetic Solids (Elsevier, 2013).

[74] G. A. Maugin, K-L: From "Kelvin Continuum" to "Long-Range Interactions", in: Non-Classical Continuum Mechanics, (Springer, 2017), pp. 127-148.

[75] C. Møller, The theory of relativity, International series of monographs on physics (Clarendon Press, 1972).

[76] R. Netz, The Works of Archimedes: Volume 1, The Two Books On the Sphere and the Cylinder: Translation and Commentary, The Works of Archimedes (Cambridge University Press, Camebridge, 2004).

[77] R. Netz and W. Noel, The Archimedes Codex: Revealing The Secrets Of The World's Greatest Palimpsest (Hachette UK, 2011).

[78] W. Noll, Euclidean geometry and Minkowskian chronometry, The American Mathematical Monthly 71(2), 129-144 (1964).

[79] W. R. Paton, Polybius. The Histories, No. 1 in The Loeb Classical Library (Harvard University Press, Cambridge, MA, 1922).

[80] G. Piola, Sull'Applicazione de' Principj della Meccanica Analitica del Lagrange ai Principali Problemi (Imp. R. Stamperia, Milano, 1825).

[81] G. Piola, La Meccanica de' Corpi Naturalmente Estesi: Trattata col Calcolo delle Variazioni (Presso Paolo Emilio Giusti, Milano, 1833).

[82] G. Piola, Intorno alle equazioni fondamentali del movimento di corpi qualsivogliono considerati secondo la naturale loro forma e costituzione, Memorie di matematica e di fisica della Società italiana delle scienze 24, 1-186 (1848).

[83] G. Piola, Di un principio controverso della meccanica analitica di Lagrange e delle sue molteplici applicazioni, Memorie dell'I.R. Istituto Lombardo di scienze, lettere ed arti 6, 389-496 (1856).

[84] L. Placidi, A variational approach for a nonlinear 1-dimensional second gradient continuum damage model, Continuum Mech. Therm. 27(4), 623-638 (2015).

[85] L. Placidi, A variational approach for a nonlinear one-dimensional damage-elasto-plastic second-gradient continuum model, Continuum Mech. Therm. 28(1), 119-137 (2016).

[86] L. Placidi, E. Barchiesi, E. Turco, and N. L. Rizzi, A review on 2D models for the description of pantographic fabrics, Z. Angew. Math. Mech. 67(5), 121 (2016).

[87] L. Placidi, I. Giorgio, A. Della Corte, and D. Scerrato, Euromech 563 Cisterna di Latina 17-21 march 2014 Generalized continua and their applications to the design of composites and metamaterials: A review of presentations and discussions, Mathematics and Mechanics of Solids pp. 1-14 (2015).

[88] L. Placidi, L. Greco, S. Bucci, E. Turco, and N. L. Rizzi, A second gradient formulation for a 2D fabric sheet with inextensible fibres, Z. Angew. Math. Mech. 67(5), 114 (2016).

[89] P. Podio-Guidugli, A virtual power format for thermomechanics, Continuum Mech. Therm. 20(8), 479-487 (2009).

[90] P. Podio-Guidugli and M. Vianello, Hypertractions and hyperstresses convey the same mechanical information, Continuum Mechanics and Thermodynamics 22(3), 163-176 (2010). 
[91] R. Prud'homme and R. Gatignol, Thick fluid interface modeling, Int. J. Therm. Sci. 43(4), 339-345 (2004).

[92] V. Rose, Archimedes im Jahre 1269, Deutsche Litteraturzeitung (1884), cc. 210-13.

[93] L. Russo, The forgotten revolution: How science was born in 300 BC and why it had to be reborn (Springer, New York, 2004).

[94] P. Seppecher, Thermodynamique des zones capillaires, in: Ann Phys-Paris, (1988), pp. 13-22.

[95] P. Seppecher, Equilibrium of a Cahn-Hilliard fluid on a wall: influence of the wetting properties of the fluid upon the stability of a thin liquid film, Eur. J. Mech. B-Fluid 12, 69-69 (1993).

[96] L. Söderholm, A principle of objectivity for relativistic continuum mechanics, Archive for Rational Mechanics and Analysis 39(2), 89-107 (1970).

[97] E. W. Sutton and H. Rackham, Cicero. On the Orator: Books 1-2, Loeb Classical Library (Harvard University Press, Cambridge, MA, 1942).

[98] A. H. Taub, Relativistic fluid mechanics, Annual review of fluid mechanics 10(1), 301-332 (1978).

[99] I. Thomas, Greek Mathematical Works, Volume II: Aristarchus to Pappus, Loeb Classical Library (Harvard University Press, Cambridge, MA, 1941).

[100] R. A. Toupin, Elastic materials with couple-stresses, Arch. Ration. Mech. An. 11, 385-414 (1962), 10.1007/BF00253945.

[101] G. W. Trompf, The Idea of Historical Recurrence in Western Thought: From Antiquity to the Reformation (University of California Press, 1979).

[102] G. W. Trompf, Vico's universe. La provvedenza and la poesia in the new science of Giambattista Vico, Br. J. Hist. Philos. 2(1), 55-86 (1994).

[103] C. Truesdell, A First Course in Rational Continuum Mechanics (Academic Press, 1977).

[104] C. Truesdell, An Idiot's Fugitive Essays on Science: Methods, Criticism, Training, Circumstances, (Springer-Verlag, New York, 1984), 1984, chap. The computer: ruin of science and threat to mankind, pp. 594-631.

[105] C. Truesdell and W. Noll, The non-linear field theories of mechanics, in: The non-linear field theories of mechanics, edited by S. Flügge, Encyclopedia of Physics Vol. III/3 (Springer, 1965).

[106] C. Truesdell and R. Toupin, The classical field theories, in: Principles of Classical Mechanics and Field Theory, edited by S. Flügge, Encyclopedia of Physics Vol. III/1 (Springer, 1960).

[107] E. Turco, K. Barcz, M. Pawlikowski, and N. L. Rizzi, Non-standard coupled extensional and bending bias tests for planar pantographic lattices. part I: numerical simulations, Z. Angew. Math. Phys. 67(5), 122 (2016).

[108] E. Turco, K. Barcz, and N. L. Rizzi, Non-standard coupled extensional and bending bias tests for planar pantographic lattices. part II: comparison with experimental evidence, Z. Angew. Math. Phys. 67(5), 123 (2016).

[109] E. Turco, F. dell'Isola, N. L. Rizzi, R. Grygoruk, W. H. Müller, and C. Liebold, Fiber rupture in sheared planar pantographic sheets: Numerical and experimental evidence, Mech. Res. Commun. 76, 86 - 90 (2016).

[110] E. Turco, M. Golaszewski, A. Cazzani, and N. L. Rizzi, Large deformations induced in planar pantographic sheets by loads applied on fibers: Experimental validation of a discrete lagrangian model, Mech. Res. Commun. 76, 51 - 56 (2016).

[111] E. Turco and N. L. Rizzi, Pantographic structures presenting statistically distributed defects: Numerical investigations of the effects on deformation fields, Mech. Res. Commun. 77, 65 - 69 (2016).

[112] G. Vailati, Il principio dei lavori virtuali da aristotele a erone d'alessandria, nota del dott. giovanni vailati, Atti della R. Accedemia delle Scienze di Torino 32 (1897).

[113] H. von Staden, Herophilus: The Art of Medicine in Early Alexandria: Edition, Translation and Essays (Cambridge University Press, 1989). 